\title{
Plasmacytoid dendritic cells protect against immune- mediated acute liver injury via IL-35
}

\author{
Yuzo Koda, ${ }^{1,2}$ Nobuhiro Nakamoto, ${ }^{1}$ Po-Sung Chu, ${ }^{1}$ Aya Ugamura, ${ }^{1}$ Yohei Mikami, ${ }^{1}$ Toshiaki Teratani, ${ }^{1}$ Hanako Tsujikawa, ${ }^{3}$ \\ Shunsuke Shiba, ${ }^{1}$ Nobuhito Taniki, ${ }^{1}$ Tomohisa Sujino, ${ }^{1}$ Kentaro Miyamoto,, Takahiro Suzuki, ${ }^{1}$ Akihiro Yamaguchi, ${ }^{1}$ Rei Morikawa, \\ Katsuaki Sato, ${ }^{4}$ Michiie Sakamoto, ${ }^{3}$ Takayuki Yoshimoto, ${ }^{5}$ and Takanori Kanai ${ }^{1,6}$ \\ 'Division of Castroenterology and Hepatology, Department of Internal Medicine, Keio University School of Medicine, Tokyo, Japan. ${ }^{2}$ Mitsubishi Tanabe Pharma Corporation, Kanagawa, Japan. ${ }^{3}$ Department \\ of Pathology, Keio University School of Medicine, Tokyo, Japan. “ Division of Immunology, Department of Infectious Diseases, Faculty of Medicine, University of Miyazaki, Miyazaki, Japan. ${ }^{5}$ Department of \\ Immunoregulation, Institute of Medical Science, Tokyo Medical University, Tokyo, Japan. ${ }^{6}$ Japan Agency for Medical Research and Development (AMED), Tokyo, Japan.
}

\begin{abstract}
Acute liver failure (ALF) is a life-threatening condition, and liver transplantation is the only therapeutic option. Although immune dysregulation is central to its pathogenesis, the precise mechanism remains unclear. Here, we show that the number of peripheral and hepatic plasmacytoid DCs (pDCs) decrease during acute liver injury in both humans and mice. Selective depletion of pDCs in Siglech ${ }^{\text {dtr/+ }}$ mice exacerbated concanavalin A-induced acute liver injury. In contrast, adoptively transferred BM-derived pDCs preferentially accumulated in the inflamed liver and protected against liver injury. This protective effect was independent of TLR7 and TLR9 signaling, since a similar effect occurred following transfer of MyD88-deficient pDCs. Alternatively, we found an unexpected immunosuppressive role of pDCs in an IL-35-dependent manner. Both I/12a and Ebi3, heterodimeric components of IL-35, were highly expressed in transferred pDCs and CD4+CD25+ Tregs. However, the protective effect of pDC transfer was completely lost in mice depleted of Tregs by anti-CD25 antibody. Moreover, pDCs derived from IL-35-deficient mice had less of a protective effect both in vivo and in vitro even in the presence of Tregs. These results highlight a unique aspect of pDCs in association with Tregs, serving as a guide for immunotherapeutic options in ALF.
\end{abstract}

\section{Introduction}

Acute liver failure (ALF) is a life-threatening condition characterized by progressive and extensive multilobular damage of the hepatocytes and massive intrahepatic infiltration of immune cells. However, there are no definitive therapeutic options for ALF other than liver transplantation, and the increasing demand for liver transplantation and insufficient numbers of donor organs highlight the need for finding alternative therapies for this condition.

Immune dysregulation plays a central role in the pathogenesis of ALF $(1,2)$. The initial massive death of hepatocytes results in marked activation of the innate immune responses (immune active phase), which is accompanied by a compensatory antiinflammatory response in parallel to or in the late phase of immune activation (immune-tolerant phase), leading to hepatic repair. Thus, it is critical to understand the underlying mechanism of immune dysregulation based on the immune phase during ALF to achieve appropriate clinical intervention. Various immune components, including Th1/Th17 cells, CD $4^{+} \mathrm{CD} 25^{+}$Tregs, NK cells, NKT cells, macrophages (Kupffer cells), conventional or classical DCs (cDCs), and plasmacytoid DCs (pDCs) reside in the liver and participate in balancing the local immune response (3-6). It is generally accepted that an imbalance between effec-

Conflict of interest: The authors have declared that no conflict of interest exists. Copyright: (5) 2019, American Society for Clinical Investigation.

Submitted: October 29, 2018; Accepted: May 14, 2019; Published: July 2, 2019.

Reference information: / Clin Invest. 2019;129(8):3201-3213.

https://doi.org/10.1172/JCl125863. tor and regulatory cells determines the prognosis during acute inflammation. Thus, it is clinically relevant to supply immunoregulatory cells such as Tregs in the immune-active phase during ALF; however, there is no definitive clinical evidence to specify the ideal immune cell subsets for this purpose, which is in part due to the difficulty of their efficient delivery and stable maintenance in the inflamed liver.

pDCs are a unique DC subset that specialize in the production of type 1 IFNs in a TLR7/9-dependent manner (7, 8). pDCs promote antiviral immune responses and have been implicated in the pathogenesis of autoimmune diseases (9). Indeed, the pathogenic role of pDCs through TLR7/9-IFN- $\alpha / \beta$ signaling in the liver has been demonstrated in several models to date (1012). Moreover, pDCs can induce tolerogenic immune responses by IL-10 secretion and interaction with Tregs in a TLRindependent manner $(13,14)$, suggesting their multifaceted characteristics according to tissue as well as stimulus specificity. However, the role of pDCs during the course of acute liver injury is still unknown.

Here, we demonstrate that acute liver inflammation gives rise to a shortage of peripheral and hepatic pDCs both in humans and in a murine model. To understand the mechanism, we utilized Siglech ${ }^{d t r /+}$ mice, in which pDCs are specifically depleted, which was accompanied by deterioration of concanavalin Ainduced (ConA-induced) immune-mediated acute liver injury. Furthermore, we confirmed that the adoptive transfer of pDCs efficiently ameliorated the acute liver injury through an IL-35dependent mechanism. This mechanistic insight may lead to a new therapeutic option for the refractory disease condition. 
A

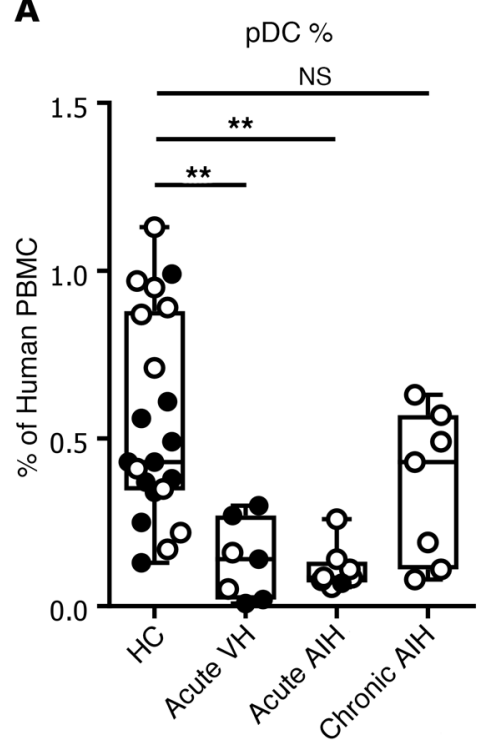

B

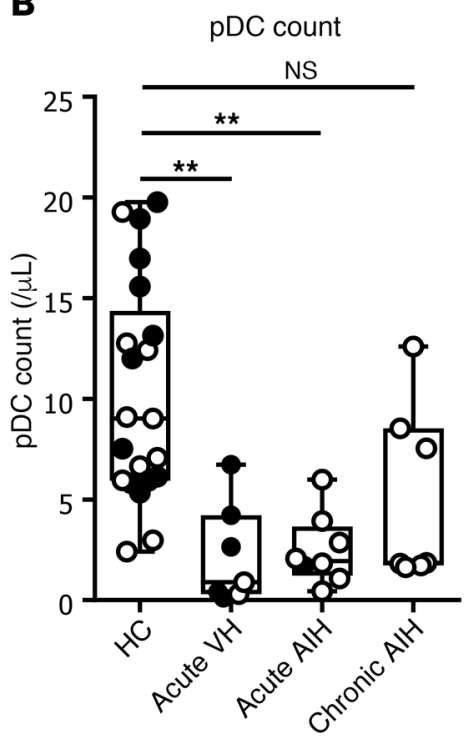

D

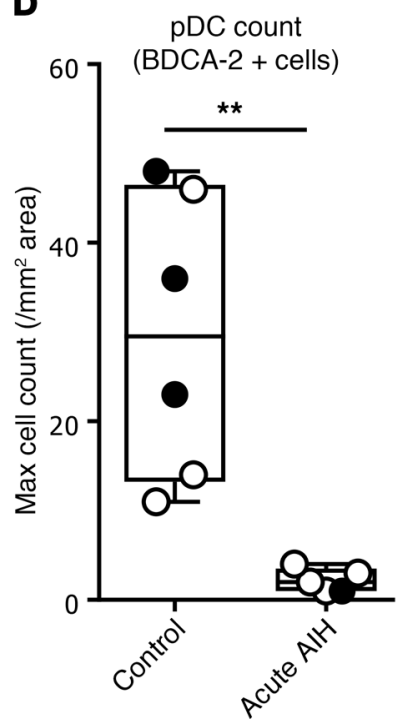

C

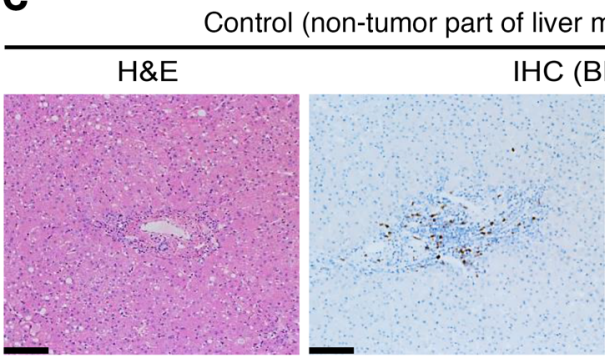

IHC (BDCA-2)

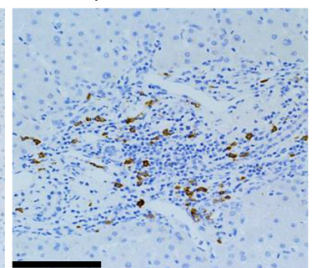

Acute $\mathrm{AlH}$

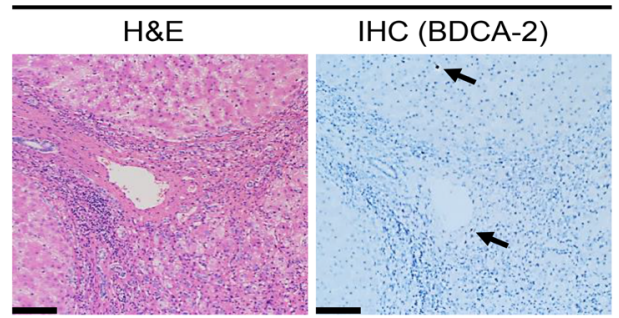

Figure 1. Reduction of pDCs in the PB and liver of acute hepatitis patients. PBMCs were analyzed in healthy control $(\mathrm{HC})(n=21)$ subjects and in patients with acute viral hepatitis (VH) $(n=7)$, acute AlH $(n=8)$, and chronic AlH $(n=7)$. (A) Mean percentages of lineage-CD123+BDCA-2+ absolute numbers in the PB. Data are presented as box-and-whisker plots. IHC was performed on liver sections of patients suffering from hepatic metastasis with normal liver function $(n=6)$ as the controls and acute AlH patients $(n=5)$. ${ }^{* *} P<0.01$, ANOVA with Tukey's multiple comparisons post-hoc test. (C) Representative photomicrographs of H\&E-stained and BDCA-2 Ab-stained IHC sections of the liver. Arrowheads indicate BDCA-2-positive cells in patients with AlH. Scale bars: $100 \mu \mathrm{m}$. (D) Maximum cell numbers per unit area $\left(1 \mathrm{~mm}^{2}\right)$. Data are presented as box-and-whisker plots. ${ }^{* *} P<0.01$, Student $t$ test. Black circles, males; white circles, females.

\section{Results}

pDCs are reduced in the liver and peripheral blood of patients with acute autoimmune hepatitis. Previous reports have shown that the frequency and number of pDCs in the peripheral blood (PB) were altered during chronic hepatitis virus infection and highly correlated with the pathology $(15,16)$. However, the role of pDCs in ALF has not been determined. Therefore, we assessed the frequency and number of peripheral pDCs in patients with acute liver injury. The clinical characteristics of healthy controls $(n=21)$ and patients with acute viral hepatitis $(n=7)$, acute autoimmune hepatitis (AIH) $(n=8)$, and chronic AIH $(n=7)$ are shown in Supplemental Table 1 (supplemental material available online with this article; https:// doi.org/10.1172/JCI125863DS1). All patients with acute AIH were newly diagnosed, and the blood samples were obtained before the initiation of immunosuppressive treatment. We defined human pDCs as lineage ${ }^{-}$CD $123^{+}$BDCA-2 ${ }^{+}$cells in this study (Supplemental Figure 1). Both the frequency and number of human peripheral pDCs were significantly reduced in acute viral hepatitis and AIH patients compared with those of the healthy control group (Figure 1, A and B, and Supplemental Figure 1). Although there were more female patients in the AIH group, this did not appear to influence the observed reduction, since there was no difference in the frequency or number of pDCs regardless of sex in healthy controls.

We further examined the involvement of pDCs in the liver during the course of acute liver injury using immunohistochemical staining of BDCA-2, a specific marker of human pDCs $(9,17)$, in liver samples obtained from patients with ALF due to acute AIH $(n=5)$ and patients suffering from hepatic metastasis of gastrointestinal cancer with normal liver function $(n=6)$ as the controls (Supplemental Table 1). Consistently, BDCA- $2^{+}$pDCs were mainly detected around the portal area in the control liver samples, whereas the number was decreased significantly in the livers of patients with ALF due to acute AIH (Figure 1, C and D), reinforcing the involvement of pDCs in the pathogenesis of acute liver injury regardless of the presence of viral infection.

pDCs are reduced in the liver and $P B$ during ConA-induced acute liver inflammation in mice. To confirm whether the findings obtained from human subjects could be recapitulated in an animal model, the dynamics of pDCs in various organs were examined during acute liver inflammation using a ConA- 
A
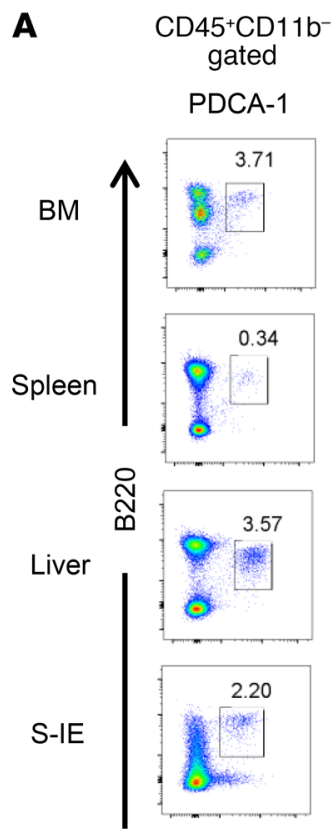

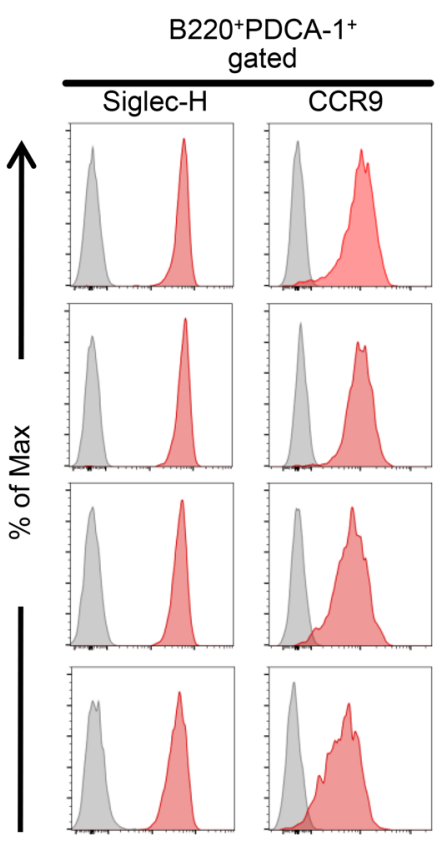

B

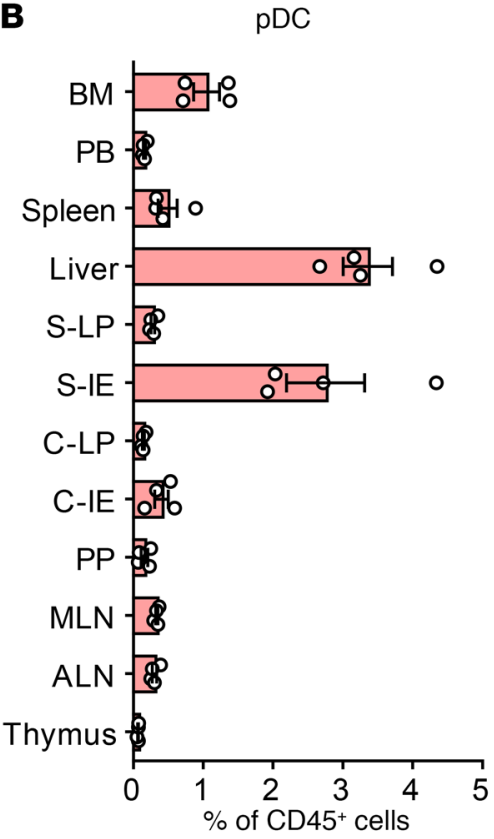

C

Liver pDC \%

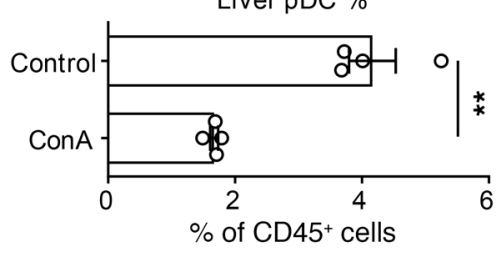

$\mathrm{PB} \mathrm{pDC} \%$
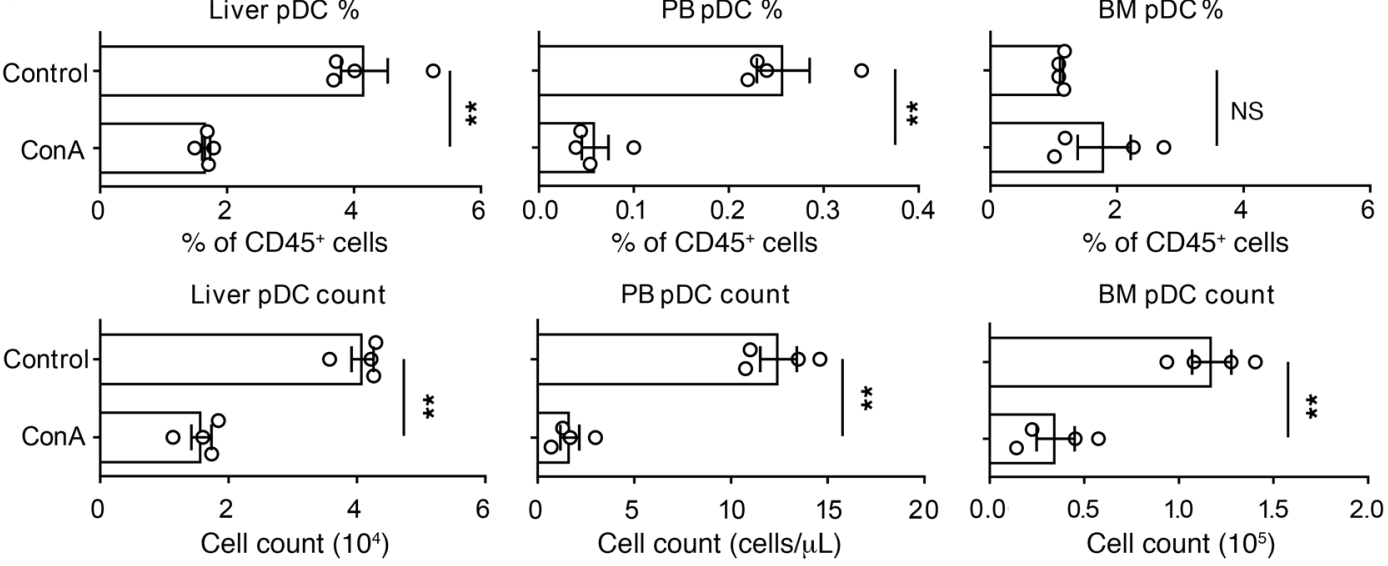

Figure 2. Reduction of pDCs in the PB and liver during ConA-induced inflammation in mice. (A) Representative B220 and PDCA-1 staining of CD45 $+C D 11 \mathrm{~b}$ -gated BM, spleen, liver, and small intestinal epithelium (S-IE) MNCs in male C57BL/6 mice (left). Representative Siglec-H (center) and CCR9 (right) histograms of $C D 45^{+} C D 11 b^{-B} 220^{+} P D C A-1^{+} p D C s$ in the BM, spleen, liver, and small intestinal epithelium. (B) Mean percentages of pDCs in CD45 $5^{+}$M, PB, spleen, liver, small-intestine lamina propria (S-LP), small intestinal epithelium, colon lamina propria (C-LP), colon intestinal epithelium (C-IE), Peyer's patches (PP), mesenteric lymph nodes (MLN), axillary lymph nodes (ALN), and thymus MNCs. Data are shown as mean \pm SEM ( $n=4$ per organ). (C) Mean percentages (upper panels) and absolute numbers (lower panels) of pDCs in CD45 liver (left), PB (center), and BM (right) MNCs of mice analyzed 18 hours following PBS (control) or ConA injection. Data are shown as mean \pm SEM $\left(n=4\right.$ per group). ${ }^{* *} P<0.01$, Student's $t$ test. Data are representative of over 3 independent experiments.

induced T cell-mediated acute liver injury mouse model. In this case, pDCs were defined as $\mathrm{CD} 45^{+} \mathrm{CD} 11 \mathrm{~b}-\mathrm{B} 220^{+} \mathrm{PDCA}-1^{+}$cells, which also highly express Siglec-H and CCR9 (Figure 2A). In the steady state, pDCs were found to be more abundant in the liver and small intestine epithelium compared with other organs (Figure 2B). However, following ConA administration, the frequency and number of pDCs in the $\mathrm{PB}$, liver, and $\mathrm{BM}$ were dramatically reduced, consistent with our previous report (Figure $2 \mathrm{C}$ and ref. 18). Furthermore, liver pDCs were prone to apoptotic cell death during ConA-induced inflammation (Supplemental Figure 2). These results, along with the findings from human samples, further support an association between the status of liver inflam- mation in the acute phase and the number of pDCs both in the liver and the periphery.

Depletion of pDCs worsens ConA-induced acute liver inflammation. Next, to clarify the role of pDCs in non-virus-mediated acute liver injury, the susceptibility to ConA-induced liver injury was compared between WT mice and mice with predepleted pDCs. We initially considered using a neutralizing Ab to PDCA-1 as a specific pDC marker in the steady state. However, PDCA-1 was also expressed in inflammatory $\mathrm{CD} 11 \mathrm{~b}^{+} \mathrm{CD} 11 \mathrm{c}^{-}$macrophages and $\mathrm{CD}_{11} \mathrm{~b}^{+} \mathrm{CD} 11 \mathrm{c}^{+} \mathrm{cDCs}$ in the mouse liver following ConA administration (Figure 3A). In contrast, Siglec-H, another pDC marker in mice $(9,19)$, was specifically expressed on pDCs in either state 
A

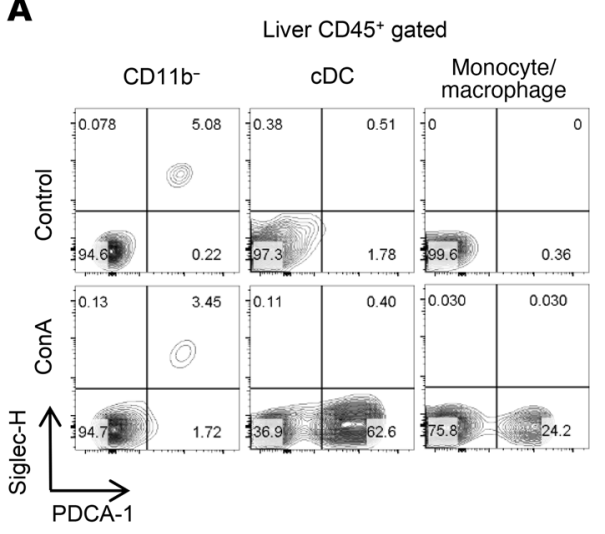

B

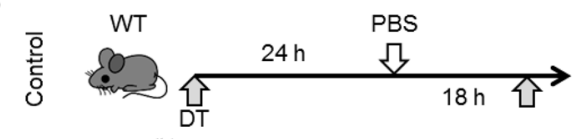

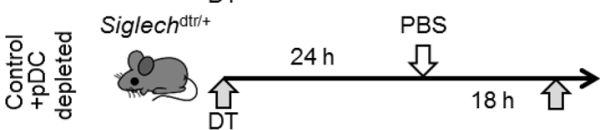

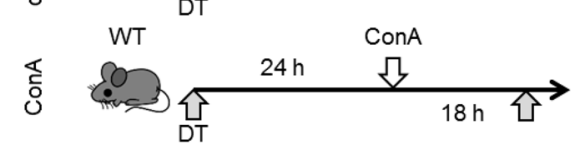

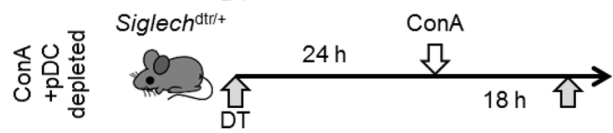

E

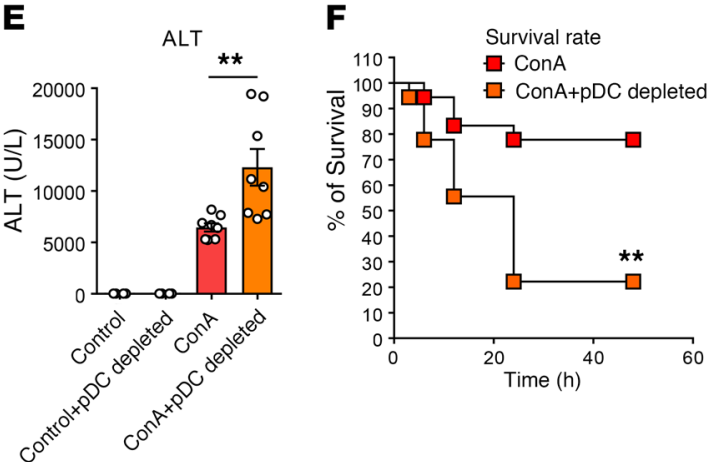

C
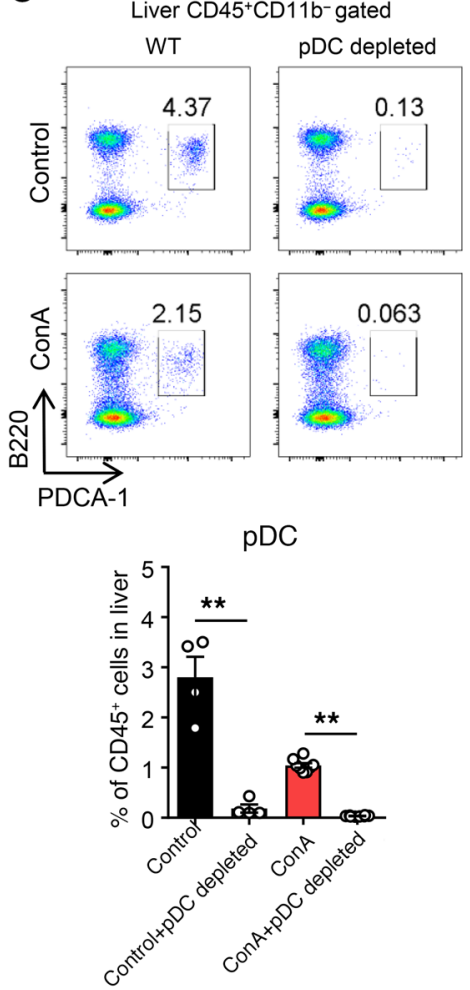

D

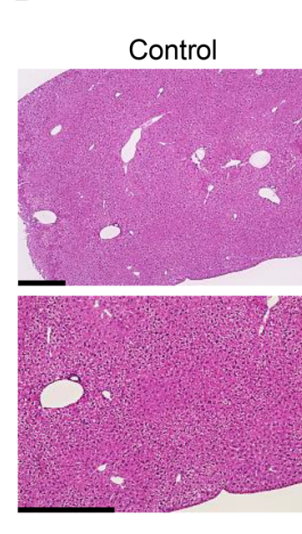

ConA
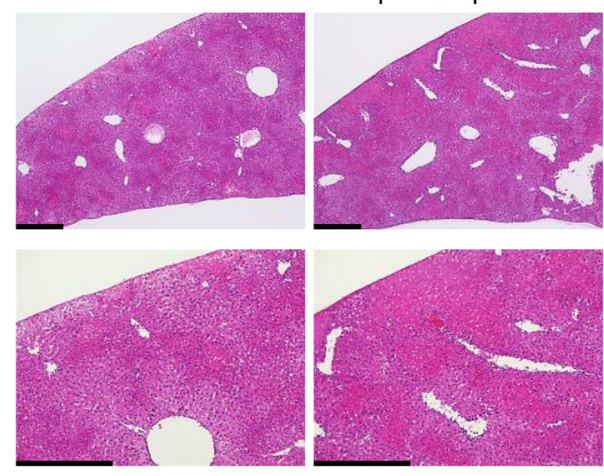

H
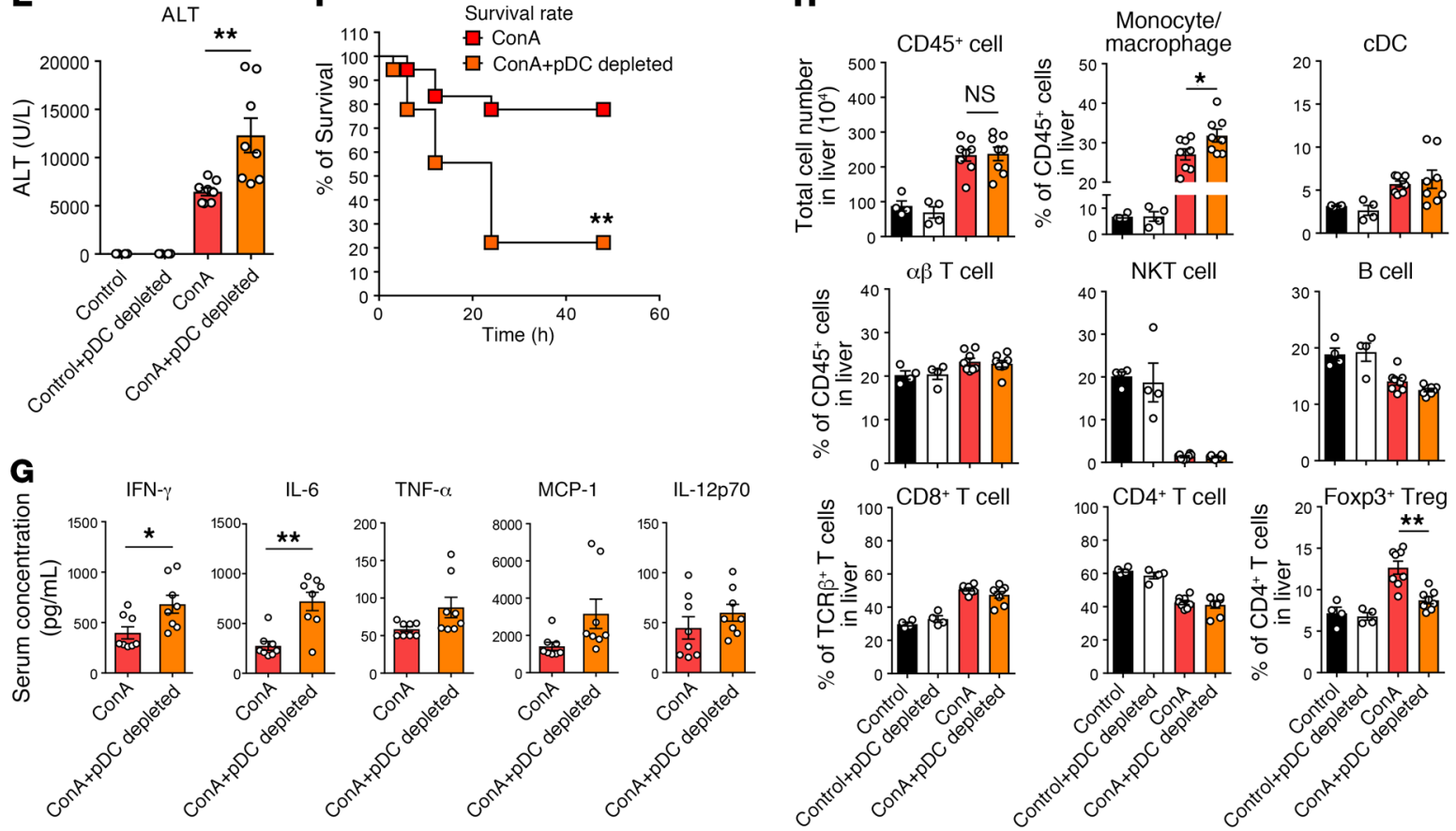
Figure 3. Siglec-H-dependent depletion of pDCs exacerbates ConAinduced inflammation. (A) Representative Siglec-H and PDCA-1 staining of CD11b cells (left), CD11b+CD11c ${ }^{+}$CDCs (center), and CD11b ${ }^{+}$CD11 $c^{-}$monocytes/macrophages (right) in the liver CD45+ MNCs. (B) Study design. WT or Siglech ${ }^{d t r /+}$ mice were treated with DT 24 hours prior to ConA (15 $\mathrm{mg} / \mathrm{kg}$ ) or PBS injection. All mice were sacrificed and analyzed 18 hours after the ConA injection. (C) Representative B220 and PDCA-1 staining of CD45 ${ }^{+}$CD11b-gated liver MNCs (left). Mean percentages of pDCs in CD45+ liver MNCs of the indicated mice (right). Data are shown as mean \pm SEM ( $n=4$ for the control or control+pDC-depleted group; $n=7$ for the ConA or ConA+pDCs-depleted group). ${ }^{* *} P<0.01$, Student's $t$ test. (D) Representative photomicrographs of $\mathrm{H} \& \mathrm{E}$-stained sections of the liver. Scale bars: $500 \mu \mathrm{m}$. (E) Serum ALT levels. (F) Survival rate. (C) Cytokine concentrations in the serum of the indicated mice. $(\mathbf{H})$ Mean percentages of various immune cells in CD45 $5^{+}$, CD45 ${ }^{+} T C R \beta^{+}$-, or CD45 $5^{+} T C R \beta^{+} C D 4^{+}$-gated liver MNCs of the indicated mice. Data are shown as mean \pm SEM $(n=4$ for the control or control $+p D C s$-depleted group; $n=7$ for the ConA or ConA+pDCs-depleted group), except in the survival assay in which $20 \mathrm{mg} / \mathrm{kg}$ ConA was injected and the data are presented as a Kaplan-Meier curve ( $n=18$ per group). ${ }^{*} P<0.05$; ${ }^{* *} P<0.01$, Student's $t$ test $(\mathbf{C}, \mathbf{E}, \mathbf{G}$, and $\mathbf{H})$ or log-rank test $(\mathbf{F})$. Data are combined from 2 independent experiments.

(Figure 3A and Supplemental Figure 3). Therefore, we applied the Siglec-H-DTR system for this examination. ConA was injected 24 hours after intraperitoneal administration of diphtheria toxin (DT) to Siglech ${ }^{d t r /+}$ and WT mice (Figure 3B), which resulted in elimination of $95 \%$ of the pDCs in both the steady state and inflammatory conditions in Siglech ${ }^{d t r /+}$ mice (Figure 3C). Importantly, pDC depletion exacerbated ConA-induced liver inflammation, as confirmed by histology, serology, and survival rate comparisons (Figure 3, D-F). Consistent with this finding, the serum levels of proinflammatory cytokines such as IFN- $\gamma$ and IL- 6 were significantly increased (Figure 3G). FACS analysis of immunocompetent cells in the liver showed an increase in $\mathrm{CD} 11 \mathrm{~b}^{+} \mathrm{CD} 11 \mathrm{c}^{-}$monocytes/macrophages and a decrease in Foxp $3^{+}$Tregs in mice with pDC depletion, while the other immune cells were not affected (Figure $3 \mathrm{H}$ ). The gating strategy of FACS analysis in this study is shown in Supplemental Figure 4. Further detailed analysis revealed that the frequency of BM-derived monocytes was increased in pDC-depleted mice, whereas no difference was observed in liver-resident macrophages and neutrophils (Supplemental Figure 5). These results suggest a protective role of $\mathrm{pDCs}$ in acute liver inflammation.

Adoptive transfer of BM-derived pDCs suppresses acute liver inflammation. To confirm the protective role of pDCs against ConA-induced acute inflammation, we established an adoptive transfer model of Flt-3L-induced pDCs derived from the BM. Murine BM cells were cultured with Flt-3L for 8 days, and then the pDCs were specifically separated (Supplemental Figure 6). Approximately $20 \%$ of the transferred pDCs derived from Ly5.1 mice migrated to the liver, which was greater than the rate of migration to the spleen, BM, and small intestine epithelium, in the steady state (Figure $4 \mathrm{~A}$ ). The transferred pDCs could also efficiently migrate to the liver during ConA-induced acute inflammation (Figure 4, B and C). As expected, the adoptive transfer of pDCs, but not cDCs, protected the mice from ConA-induced acute liver injury based on histology, serology, and survival rate comparisons (Figure 4, D-G), suggesting a specific role of pDCs. Of note, supplementation of pDCs could also protect the mice from acute liver injury even up to 8 hours following ConA administration
(Supplemental Figure 7). Consistent with these findings, serum levels of IFN- $\gamma$, IL-6, and MCP-1 were significantly decreased following pDC supplementation (Figure $4 \mathrm{H}$ ). Furthermore, the transferred pDCs suppressed the hepatic expression of IrfI and Fas and conversely enhanced the expression of anti-apoptosis-related genes acting downstream of IFN- $\gamma$ signaling (Figure 4I). The hepatoprotective effects with the regulation of immune responses by adoptive pDCs transfer were further confirmed using other acute nonviral liver inflammation models of carbon tetrachlorideinduced ( $\mathrm{CCl}_{4}$-induced) acute hepatitis and 3,5-diethoxycarbonyl-1,4-dihydrocollidine-induced (DDC-induced) cholangitis (Supplemental Figure 8).

In contrast, the adoptive transfer of Tregs derived from the spleen failed in protecting mice from ConA-induced acute liver injury (Supplemental Figure 9, A-C). Of note, the number of efficiently transferred pDCs in the inflamed liver was significantly higher than that of Tregs, even though the same number of cells were initially injected (Supplemental Figure 9, D and E). The lack of protection after Treg transfer was not considered to be due to the lack of pDCs in the liver, since simultaneous supplementation of Tregs and pDCs did not enhance the protective effect on acute liver injury and also did not influence the efficiency in the migration of Tregs to the inflamed liver (Supplemental Figure 9, F-H).

$B M$-derived pDCs induce IL-35 production via Tregs during ConA-induced acute liver inflammation. We next examined the influence of IL-10 and IL-27, given that pDCs were previously shown to regulate liver inflammation through the production of these antiinflammatory cytokines $(20,21)$. There was no change in IL-10 and IL-27 levels associated with pDC transfer (Figure 5A). However, the level of IL-35, an immune-suppressive molecule in the IL-12 family, was dramatically and specifically increased following pDC transfer (Figure 5A). Specific IL-35 neutralization with anti-IL-35 monoclonal Ab (Merck Millipore, clone V1.4C4.22) prevented the suppressive effect of pDCs on ConA-induced acute liver injury (Figure 5, B and C), hepatic Th1 and Th17 cells, and the subsequent IFN- $\gamma$ signaling (Figure 5, D-F). Collectively, these results suggest that liver inflammation was suppressed in an IL-35-dependent manner.

To clarify the mechanism of IL-35 induction, we analyzed the expression levels of IL-35-related genes in liver CD $4{ }^{+} \mathrm{CD} 25^{+}$ CD45RB ${ }^{\text {lo }}$ Tregs, which have been reported to produce IL-35 (22, 23). As shown in Figure 6A, the expression of $I l 12 a$ and Ebi3, which encode the subunits that form the IL-35 heterodimer, was significantly upregulated in liver Tregs of ConA+pDC mice, whereas there was no change in the expression of $I l 12 b$ and $I l 27 p 28$, corresponding to IL-12 and IL-27 (Figure 6A). Moreover, depletion of Tregs using anti-CD25 Ab (Bioxcell, clone PC61) (Figure 6, B-D) significantly blocked IL-35 production in the pDC-transferred mice (Figure 6E), resulting in exaggerated liver inflammation (Figure 6F). Although we could not fully exclude the possibility that anti-CD25 Ab also depleted activated $\mathrm{T}$ cells to a certain extent, these results suggest that pDCs suppress ConA-induced acute liver inflammation via IL-35 and Tregs.

$I L-35$ production from transferred pDCs contributes to suppression of ConA-induced acute liver inflammation. To elucidate the mechanism by which pDCs induce IL-35 production from Tregs, we next examined the expression of the IL-35 receptor genes Il6st 


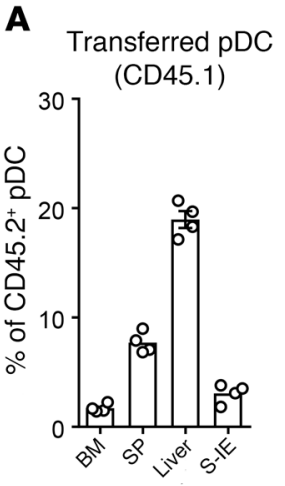

B WT (Ly5.
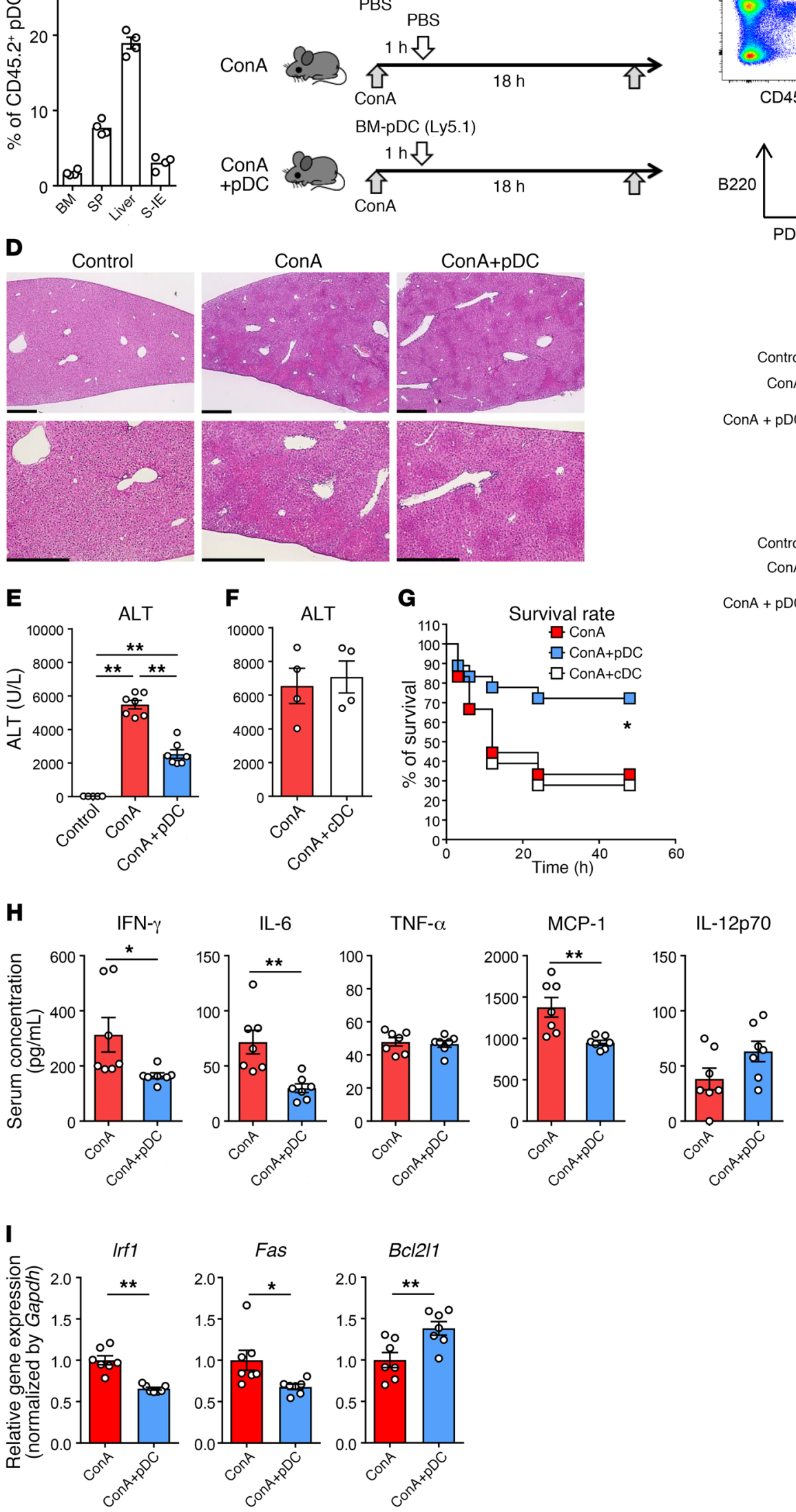

C

ConA+pDC liver CD45 ${ }^{+} C D 11 b^{-}$gated
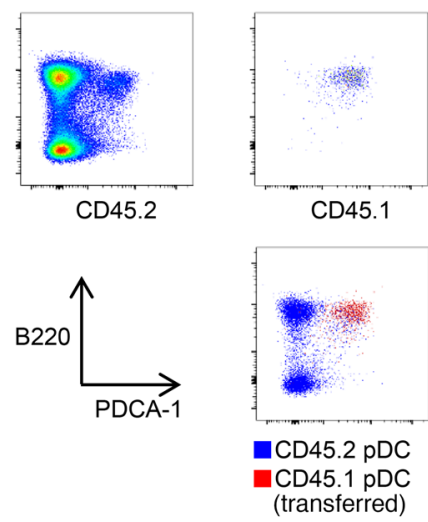

Liver pDC \%

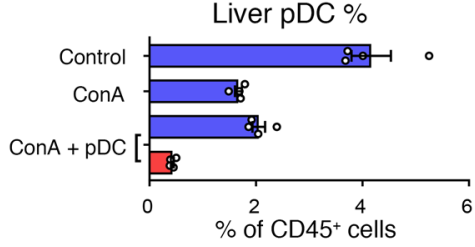

Liver pDC count

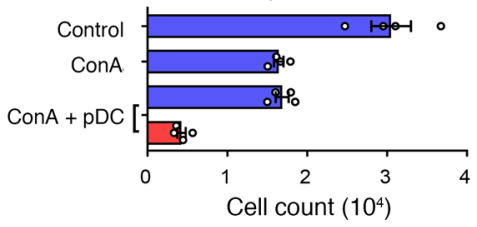

Figure 4. Adoptive transfer of BM-derived pDCs ameliorates ConA-induced inflammation. (A) Mean ratios (percentages) of transferred $B M$-derived $\mathrm{pDCs}$ (CD45.1) to intrinsic pDCs (CD45.2) in the BM, spleen, liver, and small intestinal epithelium under normal conditions. All mice were sacrificed and analyzed 24 hours after the BM-derived pDC inoculation. Data are representative of over 3 independent experiments. (B) Study design. WT (Ly5.2) mice were given intravenous injections of ConA ( $15 \mathrm{mg} /$ $\mathrm{kg}$ ) or PBS. One hour later, mice were intravenously inoculated with Flt-3L-proliferated BM-derived pDCs $\left(2 \times 10^{6}\right.$ cells $/ 200 \mu \mathrm{L}$ PBS $)$ or $200 \mu \mathrm{L}$ PBS alone. All mice were sacrificed and analyzed 18 hours after the ConA injection. (C) Representative B220 and PDCA-1 staining of CD45.2 cells (left, blue), CD45.1 cells (upper right, red), and merged cells (lower right) in CD45+CD116--gated liver MNCs of the pDC-transferred mice. Mean percentages (upper) and absolute numbers (lower) of CD45.2 pDCs (blue bars) and CD45.1 pDCs (red bars). (D) Representative photomicrographs of $\mathrm{H} \& \mathrm{E}$-stained sections of the liver. Scale bars: $500 \mu \mathrm{m}$. (E and F) Serum ALT levels. (G) Survival rates. (H) Serum cytokine concentrations. (I) IFN- $\gamma$ signaling-related gene expression levels in the whole liver. Data are shown as mean $\pm \operatorname{SEM}(n=5$ for the control group; $n=7$ for the ConA or ConA+pDC group), except for the survival assay, in which 30 $\mathrm{mg} / \mathrm{kg}$ ConA was injected and data are presented as the Kaplan-Meier curve ( $n=18$ per group). ${ }^{*} P<0.05$; ${ }^{*} P<0.01$, Student's $t$ test $(\mathbf{F}, \mathbf{H}$, and $\mathbf{I})$, ANOVA with Tukey's multiple comparisons post-hoc tests (E), or log-rank test (G). Data are combined from 2 independent experiments. 
A
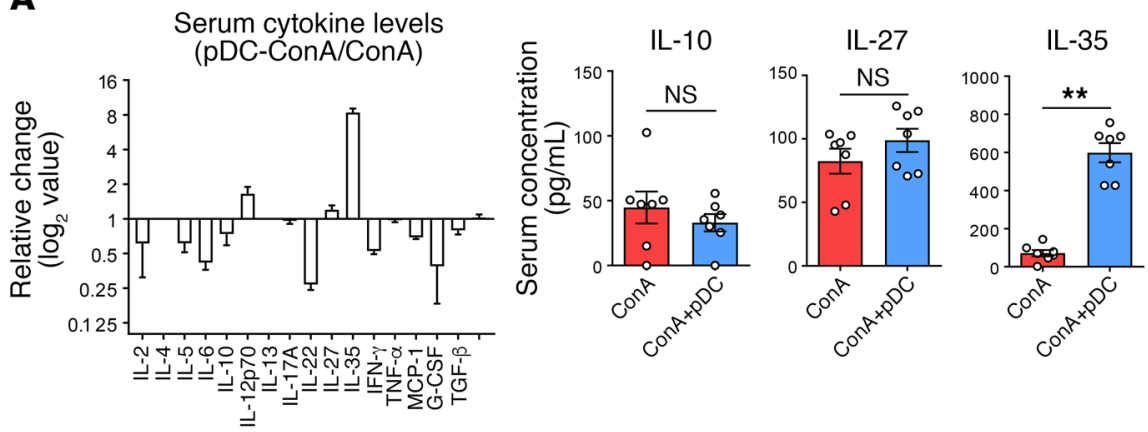

C

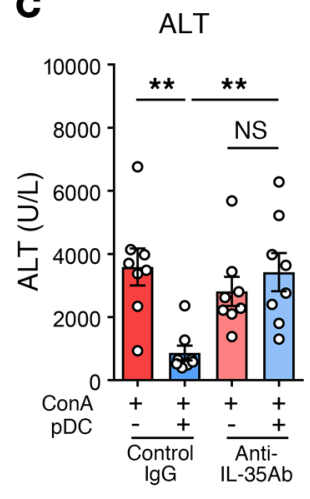

D

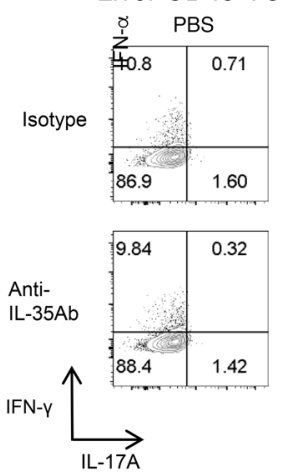

Liver CD45+TCR $\beta^{+}$CD4 ${ }^{+}$gated
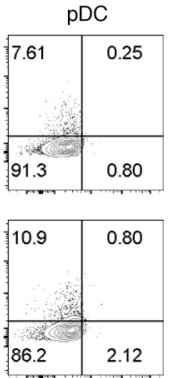

E

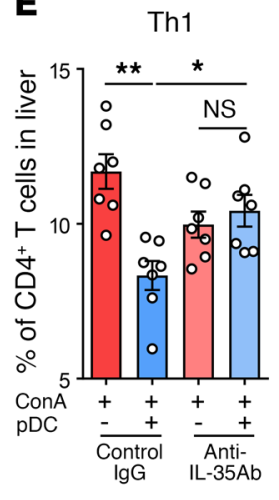

B

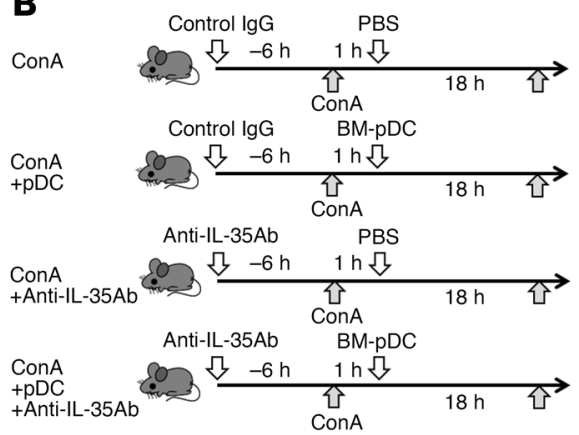

Th17

$\mathbf{F}$

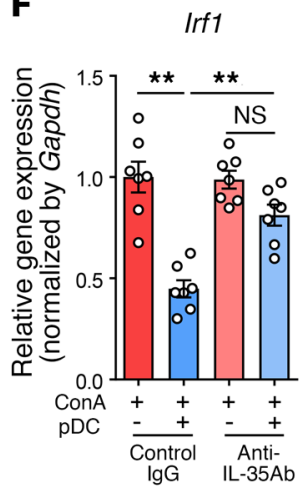

Figure 5. Suppressive effect of BM-derived pDCs on ConA-induced inflammation is dependent on IL-35. (A) Relative changes of the serum cytokine concentration in PDC-ConA-treated mice compared with ConA-treated mice. Data are shown as mean fold changes + SEM $(n=7)$ (left). Concentrations of IL-10, IL-27, and IL-35 in the serum of the indicated mice. Data are shown as mean \pm SEM ( $n=7$ for the ConA or ConA+pDC group) (right). ${ }^{* *} P<0.01$, Student's $t$ test. (B) Study design. WT mice were treated with anti-IL-35 monoclonal Ab or isotype control (200 $\mu \mathrm{g} / \mathrm{head})$ intraperitoneally 6 hours prior to ConA (15 $\mathrm{mg} / \mathrm{kg}$ ) or PBS injection. One hour later, mice were inoculated intravenously with Flt-3L-proliferated BM-derived pDCs ( $2 \times 10^{6}$ cells $/ 200 \mu \mathrm{L} \mathrm{PBS)} \mathrm{or} 200 \mu \mathrm{L}$ PBS alone. All mice were sacrificed and analyzed 18 hours after the ConA injection. (C) Serum ALT levels. Data are shown as mean \pm SEM ( $n=8$ per group). ${ }^{*} P<0.01$, ANOVA with Tukey's multiple comparisons post-hoc test. (D) Representative intracellular IFN- $\gamma$ and IL-17A staining of CD45+TCR $\beta^{+}$CD4 $4^{+}-$gated liver MNCs. (E) Mean percentages of IFN- $\gamma^{+}$(Th1) cells (left) and IL-17+ (Th17) cells (right) in CD45+TCR $\beta^{+}$CD4+-gated liver MNCs. (F) Irf1 gene expression levels in whole livers from the indicated mice. Data are shown as mean \pm SEM ( $n=7$ per group). ${ }^{*} P<0.05$; ${ }^{* *} P<0.01$, ANOVA with Tukey's multiple comparisons post-hoc test. Data are combined from 2 independent experiments.

and Il12rb2 on liver Tregs (Figure 7A). Upregulation of these genes on Tregs following ConA administration would suggest that liver Tregs act not only as IL-35 producers, but also as receivers in the pDC-transferred condition, consistent with a previous report showing that IL-35 is a self inducer (23). Alternatively, we confirmed that transferred pDCs, but not endogenous pDCs, showed significantly higher expression levels of IL-35 genes (Figure 7B). Of note, the difference was tissue specific, since BM-derived pDCs showed higher IL-35 gene expression levels regardless of in vitro proliferation rates, supporting the notion that BM-derived pDCs are a suitable candidate for adaptive cell transfer (Supplemental Figure 10). To clarify the IL-35-mediated suppressive function of pDCs, we examined the effect of BM-derived pDCs from WT, Ill2 $a^{-1-}$, or Ebi3 $3^{--}$mice on Treg-mediated suppression against the proliferation of $\mathrm{T}$ effector (Teff) cells in vitro. The production of IL-35 was induced from Tregs and pDCs, but not by Teff cells, regardless of the stimulatory state (Figure 8A). Under cocultured conditions, pDCs derived from WT mice decreased the proliferation of Teff cells regardless of the presence of Tregs, suggesting a direct effect of pDCs on Teff cells. However, both $I 112 a^{--}$and Ebi3 ${ }^{-1}$ pDCs had weaker suppressive effects than WT pDCs in the presence of Tregs, whereas only Ebi3 ${ }^{--}$pDCs had a significantly weaker suppressive effect than WT pDCs in the absence of Tregs (Figure 8, B and C), which may reflect the function of Ebi3 on IL-27 (21). These results suggest that pDCs exert their suppressive effect on Teff function in the presence of Tregs via IL-35 in vitro. To further confirm the in vitro findings of the IL-35-mediated suppressive function of $\mathrm{pDCs}$, we transferred BM-derived $\mathrm{pDCs}$ from Ill2a $a^{-/}$or Ebi3 ${ }^{-/}$mice to ConA-administered mice (Figure 8D). Of note, the suppressive effect was significantly blocked by both Il12a- and Ebi3-deficient pDCs (Figure 8E). In contrast, Il12b-, Il10-, or MyD88-deficient pDCs suppressed ConA-induced acute inflammation as efficiently as WT pDCs (Figure 8F and Supplemental Figure 11). Similarly, the hepatoprotective effect by BMderived pDCs was not affected when IL-10 signaling was blocked by anti-IL-10R Ab s(Supplemental Figure 11). These results collectively reinforce the IL-35-mediated suppressive function of pDCs in ConA-induced acute liver injury.

\section{Discussion}

pDCs play an important pathogenic role in chronic liver injury, regardless of the presence or absence of hepatitis virus infection, through the TLR7/9-IFN signaling pathway $(7,8)$. However, recent reports have demonstrated a tolerogenic characteristic of pDCs 
A

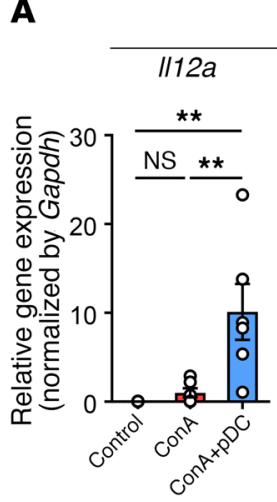

Liver Treg $\left(\mathrm{CD} 4{ }^{+} \mathrm{CD} 25^{+}{ }^{+} \mathrm{CD} 45 \mathrm{RB}^{\text {lo }}\right)$
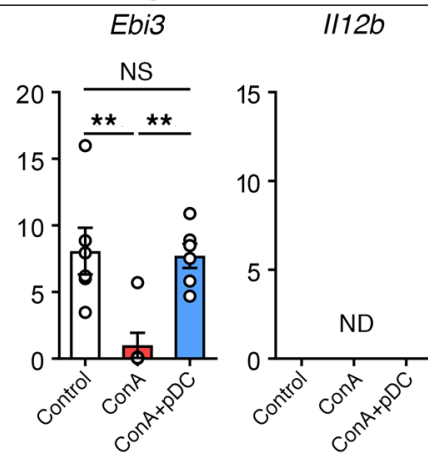

D

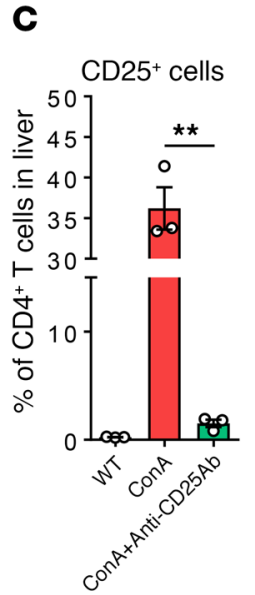

D

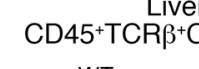

Liver
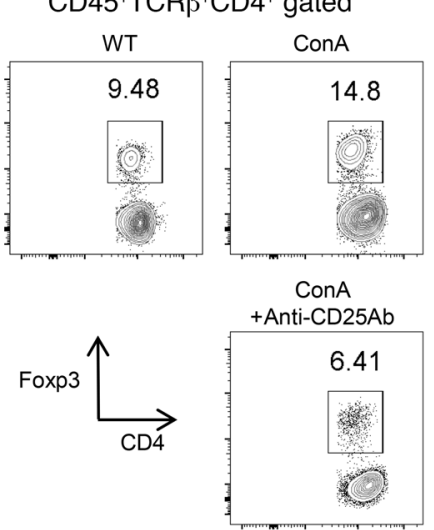
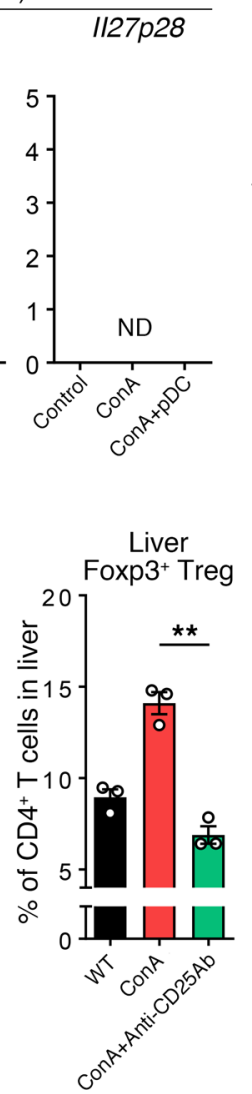

B

ConA

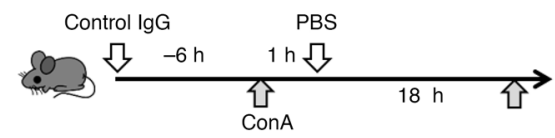

ConA

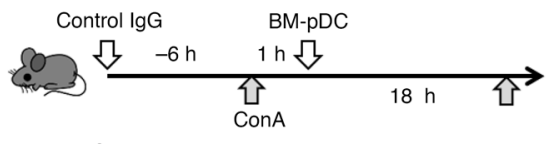

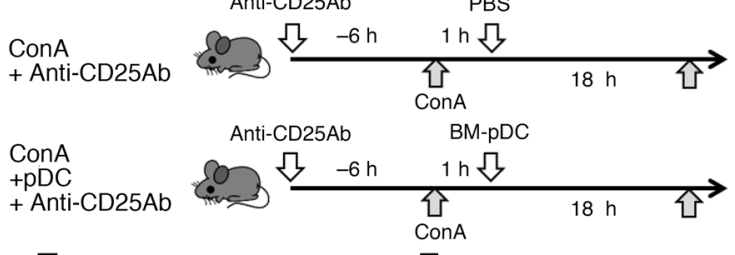

E

F

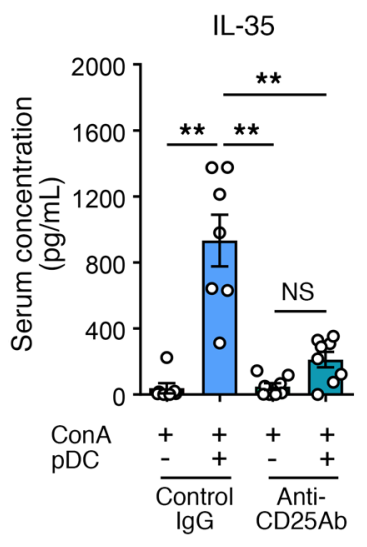

Figure 6. BM-derived pDCs ameliorate ConA-induced inflammation in the presence of IL-35-producing Tregs. (A) IL-35-related gene expression levels in liver CD45 ${ }^{+} T C R B{ }^{+} C D 4{ }^{+} C D 25^{+} C D 45 R B^{10}$ Tregs isolated from the indicated mice. Data are shown as mean $\pm \operatorname{SEM}\left(n=6\right.$ per group). ${ }^{* *} P<0.01$, ANOVA with Tukey's multiple comparisons post-hoc test. (B) Study design. WT mice were treated with anti-CD25 Ab (PC61) or isotype control (500 $\mu \mathrm{g} / \mathrm{head}$ ) intraperitoneally 6 hours prior to ConA ( $15 \mathrm{mg} / \mathrm{kg}$ ) or PBS injection. One hour later, mice were intravenously inoculated with Flt-3L-proliferated BM-derived pDCs $\left(2 \times 10^{6}\right.$ cells $/ 200 \mu \mathrm{L}$ PBS) or $200 \mu \mathrm{L}$ PBS alone. All mice were sacrificed and analyzed 18 hours after ConA injection. (C) Mean percentages of CD25+cells in $C D 45^{+} T C R \beta^{+} C D 4^{+}$-gated liver MNCs. (D) Representative Foxp3 and CD4 intracellular staining of CD45+TCR $\beta^{+}$CD4 $4^{+}$-gated liver MNCs (left) and mean percentages of Foxp $3^{+}$Tregs in CD45 ${ }^{+}$TCR $\beta^{+}$CD4 $4^{+}$-gated liver MNCs (right) of indicated mice. Data are shown as mean \pm SEM $\left(n=3\right.$ per group). ${ }^{* *} P<0.01$, ANOVA with Tukey's multiple comparisons correction. (E) Serum IL-35 concentrations of indicated mice. (F) Serum ALT levels of the indicated mice. Data are shown as mean \pm SEM ( $n=7$ for the control IgG or control IgG+pDC group; $n=8$ for the anti-CD25 Ab or anti-CD25 Ab+pDC group). ${ }^{* *} P<0.01, A N O V A$ with Tukey's multiple comparisons post-hoc test. Data are representative (C and $\mathbf{D})$ or combined $(\mathbf{A}, \mathbf{E}$, and $\mathbf{F})$ from 2 independent experiments.

in a TLR7/9-independent manner using various models $(9,14$, 24, 25). These tolerogenic pDCs have been reported to express IL-10, indoleamine 2,3-dioxygenase (IDO), ICOSL, OX4OL, and programmed cell death protein 1 ligand (PDL1). However, the potential role of hepatic pDCs in the pathogenesis of ALF and the underlying mechanism remain unclear. Here, we provide what we believe is the first demonstration that the number of peripheral and hepatic pDCs decreased during the course of immunemediated acute liver injury both in mice and humans, and adoptive transfer of BM-derived pDCs preferentially accumulated in the inflamed liver to protect mice from acute liver injury. Overall, these results highlight immunosuppressive pDCs as a potential therapeutic target.

We initially found that the numbers of circulating and hepatic pDCs were dramatically decreased in the early phase of liver injury. Loss of production in the BM (Figure 2C) or accelerated cell death in the inflamed liver (Supplemental Figure 2) could account for this decrease, consistent with previous reports demonstrating similar effects during viral infections (26). Of note, supplementation of BM-derived pDCs derived from WT or $M y D 88^{-/}$mice achieved a protective effect in ConA-induced acute liver injury, underscoring the protective role of pDCs in a TLR7/9-independent manner in this model. Instead, we here propose that IL-35-producing pDCs play an immunosuppressive role though interaction with Tregs in the liver, leading to attenuation of the acute liver injury.

IL-35, composed of the subunits IL-12a chain p35 and IL-27b $\mathrm{Ebi3}$, is a recently described heterodimeric cytokine that belongs to the IL-12 family (27). Early studies indicated a key role of IL-35 in the suppressive activity of Tregs and its contribution in the pathogenesis of allergic and autoimmune diseases $(23,28,29)$. Subsequent studies demonstrated that IL- 35 can also be produced by other cell types, such as regulatory B cells, DCs, and macrophages, in addition to Tregs (30-33). Given that both Il12a and Ebi3, but not IL-12b or IL-27, were preferentially expressed by Tregs in the livers of mice transferred with pDCs, and depletion of Tregs abrogated the protective effect of pDCs along with causing a 
A

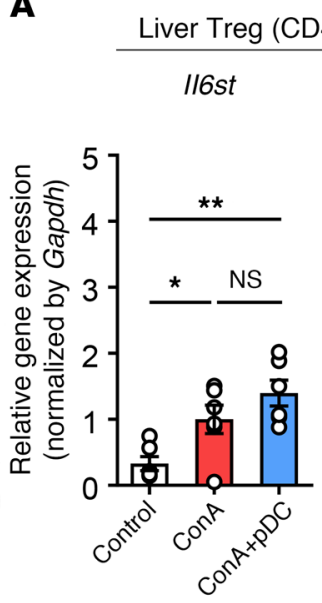

/I12rb1

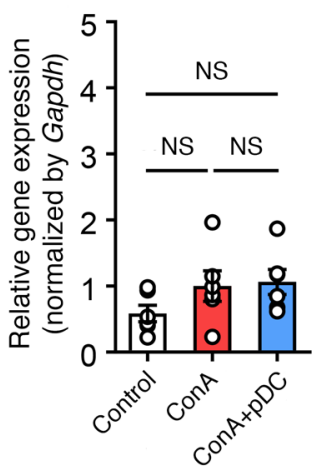

B
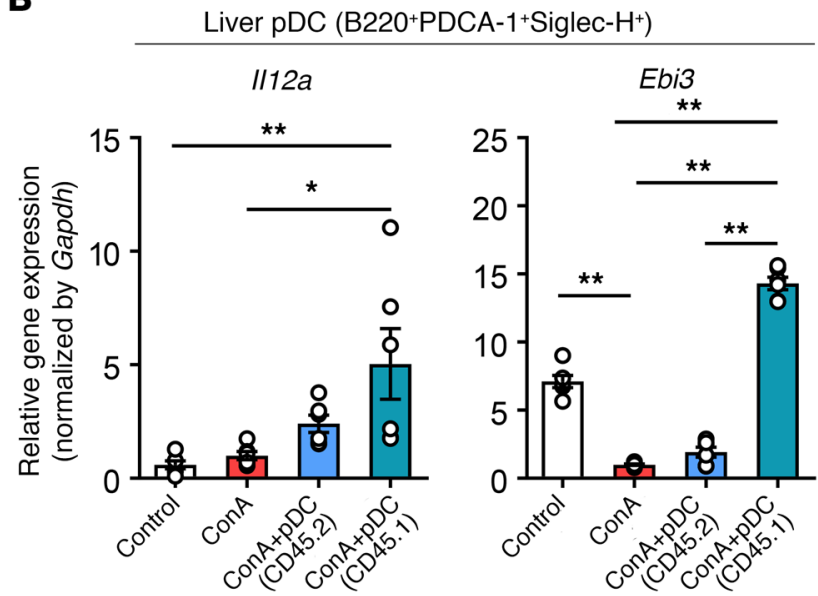

$1 / 12 b$

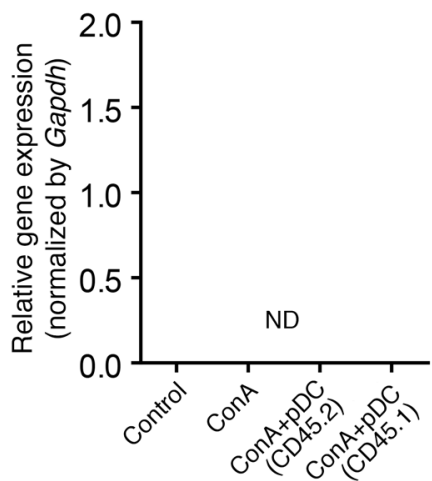

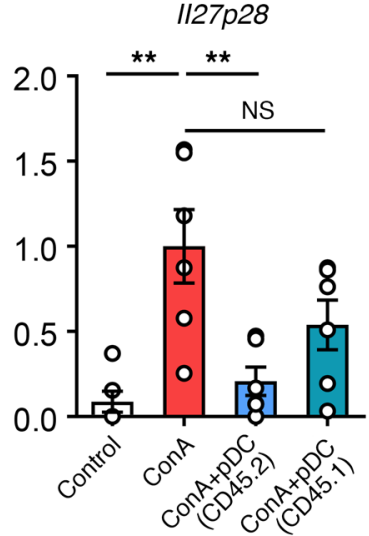

Figure 7. Transferred BM-derived pDCs express IL-35 genes during ConA-induced inflammation. (A) IL-35 receptor-related gene expression levels in liver

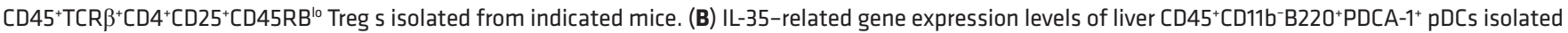
from indicated mice. Data are shown as mean \pm SEM ( $n=6$ per group). ${ }^{*} P<0.05 ;{ }^{* *} P<0.01$, ANOVA with Tukey's multiple comparisons post-hoc test. Data are combined from 2 independent experiments.

diminished serum IL-35 level, it is tempting to speculate that IL-35 directly affects Tregs in an autocrine manner; however, the details of the interaction between transferred pDCs and Tregs in the liver remain to be elucidated. One possible explanation could be related to the high expression of IL-35 receptors (Il6st and Il12rb2) in hepatic Tregs of the mice during inflammation. Moreover, transferred pDCs, but not resident pDCs, in the liver expressed higher levels of IL-35, suggesting that these cells serve as an alternative cell subset that potentially produces IL-35. Notably, pDCs derived from $\mathrm{Il} 12 \mathrm{a}^{-/-}$or $\mathrm{Ebi3}^{-/-}$mice, but not from $\mathrm{Il1O}^{-/-}$mice, demonstrated a weaker protective effect even in the presence of Tregs, further underscoring the IL-35-dependent protective effect by pDCs in association with Tregs in the liver.

We further provide insight into the mechanism by which supplementation of pDCs eventually improved ConA-induced acute liver injury, given that Th1 responses and IFN- $\gamma$ signalingassociated apoptosis in the liver were abrogated by pDCs in an IL-35-dependent manner. Furthermore, it is important to note that an increase in the number of Tregs was found in the inflamed livers of all 3 models employed in this study, suggesting that sufficient Tregs might be needed to achieve the suppressive effect by supplementation of pDCs. Destruction of the liver paren- chyma by cellular immune responses is a pathophysiologically important feature of human ALF, including acute-onset AIH (34, 35). Although the murine model used in this study does not fully mimic the human disease condition, these results along with the consistent kinetics of pDCs in human and murine livers suggest that supplementation of deficient pDCs during the course of immune-mediated ALF might be a therapeutic option.

It is widely accepted that pDCs are more abundant in the liver compared with other organs $(36,37)$, consistent with our results. This might be related to the constant exposure to various environmental factors, including microbial products, that enter the liver through the portal vein or bile ducts. However, we believe that this scenario is unlikely in our model, since the number of hepatic pDCs in the steady state was not affected in germ-free mice, gut-sterilized mice, or $M y D 88^{-/-}$ mice (data not shown). In addition, we demonstrated that the tolerogenic function of hepatic pDCs regarding IL-35 productivity differed from that of BM-derived pDCs. Since pDCs differentiate in the BM and are localized in the organs through the blood flow, it is speculated that the long-term localization in the liver might affect the phenotype and function of pDCs. However, further studies are needed to identify 

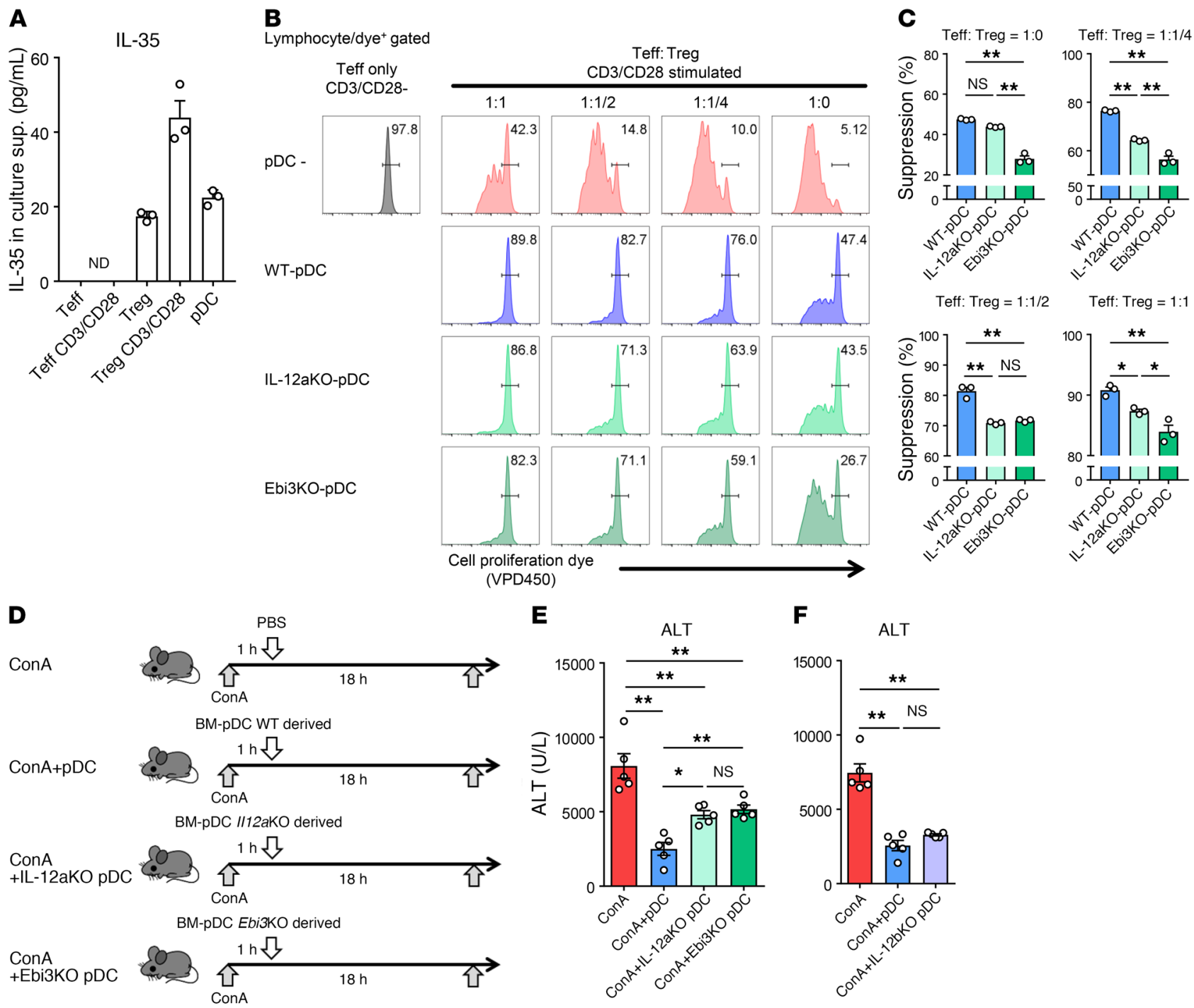

Figure 8. IL-35 production by pDCs participates in amelioration of ConA-induced inflammation. (A) IL-35 concentration in culture supernatants of spleen-derived CD4+CD25- Teff cells, CD4+CD25+ Tregs, and Flt-3L-proliferated pDCs $\left(5 \times 10^{5}\right.$ cells/well) for 24 hours in vitro. Data are shown as mean + SEM ( $n=3$ per group). (B and C) FIt-3L-proliferated pDCs derived from WT, $I 112 a^{-/}$, or Ebi3 $3^{-/-}$mice $\left(5 \times 10^{4}\right.$ cells/well) were cocultured with cell proliferation dye-stained Teff cells ( $5 \times 10^{4}$ cells/well) stimulated by CD3/CD28 microbeads in the presence of various numbers of Tregs for 4 days. Representative histograms of Teff cells (B) and suppression rate of the proliferation of Teff cells by the indicated pDCs (C). Data are shown as mean + SEM ( $n=3$ per group). ${ }^{*} P<0.05 ;{ }^{*} P<0.01$, ANOVA with Tukey's multiple comparisons post-hoc test. (D) Study design. WT mice were treated with ConA (15 mg/kg) or PBS injection. One hour later, mice were intravenously inoculated with Flt-3L-proliferated BM-derived pDCs derived from WT, $1 / 12 a^{-/}$, or Ebi3 ${ }^{-/-}$mice $(2 \times$ $10^{6}$ cells $/ 200 \mu \mathrm{L}$ PBS) or $200 \mu \mathrm{L}$ PBS alone. All mice were sacrificed and analyzed 18 hours after the ConA injection. (E and F) Serum ALT levels. Data are shown as mean \pm SEM ( $n=5$ per group). ${ }^{*} P<0.05$; ${ }^{*} P<0.01$, ANOVA with Tukey's multiple comparisons post-hoc test. Data are representative (A-C) or combined ( $E$ and $\mathbf{F}$ ) from 2 independent experiments.

and confirm the precise mechanistic factors responsible for the migration and maintenance of pDCs in the liver.

It is important to note that the adoptive transfer of Tregs was insufficient to protect mice from ConA-induced acute liver injury in this study, which conflicts with the results of a previous report (38). It is possible that the splenic Tregs derived from WT mice in this study were functionally immature compared with the hepatic pDCs derived from ConA-administered mice utilized in the previous study. Moreover, the route of cell transfer (direct injection to the liver vs. intravenous injection) might explain this difference, especially in the migration efficiency.
We also confirmed that the lack of protection after Treg transfer was not due to the lack of pDCs in the liver, since supplementation of both Tregs and pDCs did not enhance the protective effect on acute liver injury by the single supplementation of pDCs. Considering the urgent condition in patients suffering from ALF, it is critical to obtain and deliver the targeted cell subsets to the inflamed liver safely and efficiently. To overcome this potential flaw in Tregs, our results demonstrate that in vitro-expanded BM-derived pDCs could be a useful clinical option, given the potent cell transfer efficiency to the liver. The protective effect conferred by adoptive transfer of pDCs 
delivered at a later stage following ConA administration provides further support for the translational potential.

In summary, these results highlight a previously unraveled protective aspect of pDCs in association with Tregs in an IL-35dependent manner. Further research to validate and explore these findings can offer important insight for developments of new therapeutic strategies to treat ALF focusing on the manipulation of Treg activity.

\section{Methods}

Patients. PB samples were obtained from healthy controls $(n=21)$, patients with acute viral hepatitis $(n=7)$, and patients with acute AIH $(n=8)$ or chronic AIH $(n=7)$. Acute viral hepatitis was diagnosed (or excluded) serologically. Liver tissue samples were obtained from patients with ALF due to acute $\operatorname{AIH}(n=5)$ and patients suffering from hepatic metastasis of gastrointestinal cancer with normal liver function $(n=6)$ as the control group. AIH was diagnosed according to International Autoimmune Hepatitis Group (IAIHG) criteria (39). Cases of acute or chronic AIH were differentiated by the existence of a previous history of liver dysfunction and an on-going clinical course, as suggested previously $(40,41)$. The background clinical characteristics are summarized in Supplemental Table 1.

Isolation of human PBMCs. Human PBMCs were isolated by density centrifugation. Human blood was collected into a BD Vacutainer $\mathrm{CPT}$ and centrifuged at $470 \mathrm{~g}$ for 20 minutes at room temperature. PBMCs were collected at the interphase, washed, and resuspended in FACS buffer.

IHC of human liver tissues. IHC was performed using a Bond-Max automated immunohistochemical staining machine (Leica Microsystems). Anti-human CD303 (BDCA-2) monoclonal Ab (Dendritics, clone 124B3.13) was used as primary Ab. To quantify BDCA-2-positive cells, we checked all portal areas where pDCs were abundant in each section, and the highest number in $1 \mathrm{~mm}^{2}$ areas was determined as the maximum cell count.

Mice. C57BL/6 mice were purchased from Japan CLEA. Siglech ${ }^{d t r / d t r}$ mice on a C57BL/6 background were established previously (42). C57BL/6-Ly5.1 mice were obtained from Taconic Laboratory. MyD88-1mice on a C57BL/6 background were described previously (43). $\mathrm{Il1O}^{-/-}$ mice were provided by Kenya Honda (Keio University). These mice were maintained under specific pathogen-free (SPF) conditions in the Animal Care Facility of Keio University School of Medicine. $I l 12 a^{-/}, I l 12 b^{-/}$, and $\mathrm{Ebi3}^{-/-}$mice on a C57BL/6 background were obtained from Jackson Laboratory and maintained under SPF conditions in the Laboratory Animal Center of Tokyo Medical University.

ConA-induced hepatitis model. ConA (type IV, Sigma-Aldrich) was intravenously injected into the tail vein of mice (6- to 8-week-old males) at 15,20 , or $30 \mathrm{mg} / \mathrm{kg} 18$ hours or 48 hours before the study end point.

Isolation of tissue immune cells in mice. Liver mononuclear cells (MNCs) were separated from the liver as described previously with slight modifications (44). Briefly, livers were perfused through the portal vein with PBS and then minced and passed through $100 \mu$ m nylon mesh. The filtrate was centrifuged at $50 \mathrm{~g}$ for 1 minute, and the supernatant was washed once. The $\mathrm{BM}, \mathrm{PB}$, and spleen were hemolyzed and passed through the $100 \mu \mathrm{m}$ nylon mesh. Peyer's patch, mesenteric lymph nodes, axillary lymph nodes, and the thymus were minced and passed through the $100 \mu \mathrm{m}$ nylon mesh. The intestinal epithelium fraction was prepared from intestinal tissues by digestion with HBSS (Nacalai Tesque) containing $1 \mathrm{mM}$ dithiothreitol (Sigma-Aldrich) and 5 mM EDTA (Gibco, Thermo Fisher Scientific) for 30 minutes at $37^{\circ} \mathrm{C}$. Subsequently, the lamina propria fraction was prepared by digestion with a digestion solution containing $1.5 \% \mathrm{FBS}, 1.0 \mathrm{mg} / \mathrm{mL}$ collagenase A (Roche Diagnostics $\mathrm{GmbH}$ ), and $0.1 \mathrm{mg} / \mathrm{mL}$ DNase (Sigma-Aldrich) for 30 minutes at $37^{\circ} \mathrm{C}$ as described previously (45). Cells from each tissue were suspended in $40 \%$ Percoll and overlaid on a $75 \%$ Percoll fraction. Percoll gradient separation was performed by centrifugation at $840 \mathrm{~g}$ for 20 minutes at room temperature. MNCs were collected at the interphase, washed, and resuspended in FACS buffer or RPMI1640 medium (Sigma-Aldrich) containing 10\% FBS and 1\% penicillin/ streptomycin (Nacalai Tesque).

Flow cytometry and cell sorting. After blocking with anti-FcR (CD16/32, BD Biosciences - Pharmingen) for 5 minutes, the cells were incubated with the specific fluorescence-labeled $\mathrm{Ab}$ at $4^{\circ} \mathrm{C}$ for 20 minutes, followed by permeabilization and intracellular staining with anti-Foxp3 $\mathrm{Ab}$ in the case of Treg staining. The following Abs were used: anti-human CD3\& (BD Biosciences - Pharmingen, FITC, clone UCHT1), anti-human CD14 (BioLegend, PE-Cy7, clone M5E2), anti-human CD16 (BD Biosciences - Pharmingen, APCCy7, clone 3G8), anti-human CD123 (BD Biosciences - Pharmingen, PerCP-Cy5.5, clone 7G3), anti-human BDCA-2 (Miltenyi, APC, clone AC144), anti-mouse CD45 (BD Horizon, BV510, clone 30-F11), antimouse CD45.1 (BD Biosciences - Pharmingen, FITC, clone A20), anti-mouse CD11b (BD Biosciences - Pharmingen, APC-Cy7, clone M1/70), anti-mouse CD11c (BD Biosciences - Pharmingen, FITC/ PE-Cy7, clone HL3), anti-mouse B220 (BioLegend, PerCP-Cy5.5, clone RA3-6B2), anti-mouse PDCA-1 (BioLegend, APC, clone 129c1), anti-mouse Siglec-H (BioLegend, PE, clone 551), anti-mouse CCR9 (eBioscience/BD Horizon, FITC/BV421, clone eBioCW1.2/CW1.2), anti-mouse TCR- $\beta$ (BD Biosciences - Pharmingen, APC, clone H57-597), anti-mouse NK1.1 (BioLegend PE-Cy7, clone PK136), anti-mouse TCR $\gamma \delta$ (BD Biosciences - Pharmingen, FITC/PE, clone GL3), anti-mouse CD19 (BD Biosciences - Pharmingen, PE, clone 1D3), anti-mouse CD8a (BD Biosciences - Pharmingen, APC-Cy7, clone 53-6.7), anti-mouse CD4 (BD Biosciences - Pharmingen/BD Horizon, FITC/BV510, clone RM4-5), anti-mouse CD25 (BD Biosciences - Pharmingen, APC-Cy7, clone PC61), anti-mouse CD25 (BD Biosciences - Pharmingen, FITC, clone 7D4), anti-mouse CD45RB (eBioscience, PE, clone C363.16A), anti-mouse Foxp3 (eBioscience, PE/PerCP-Cy5.5, clone FJK-16s), anti-mouse Ly-6C (BioLegend, PE-Cy7, clone HK1.4), anti-mouse Ly-6G (BD Biosciences Pharmingen, APC, clone 1A8), and anti-mouse CX3CR1 (BioLegend, BV421, clone SA011F11). For intracellular cytokine staining, hepatic MNCs were stimulated with PMA (50 ng/ml) and ionomycin (500 $\mathrm{ng} / \mathrm{ml}$ ) for 3 hours in the presence of GolgiStop $(10 \mu \mathrm{g} / \mathrm{ml})$, followed by surface staining, permeabilization, and intracellular staining with anti--IFN- $\gamma$ Ab (BD Biosciences - Pharmingen, PE, clone XMG1.2) and anti-IL-17A Ab (eBioscience, APC, clone eBio17B7). For cellular apoptosis staining, the Annexin V FITC Apoptosis Detection Kit (Nacalai Tesque) was used following the manufacturer's recommendation. Events were acquired with FACS Canto II (BD) and analyzed with FlowJo software (Tree Star Inc.). Cell sorting was performed using FACS Aria (BD), and over 95\% purity of sorted cells was confirmed. The gating strategy of FACS analysis used in this study is shown in Supplemental Figures 1 and 3. 
Measurement of liver injury. Serum alanine aminotransferase (ALT) levels were measured using DRI-CHEM (Fuji Film) according to the instructions provided by the manufacturer. Livers were fixed in $10 \%$ formalin and embedded in paraffin. Sections were stained with $\mathrm{H} \& \mathrm{E}$ and examined.

Cytokine quantification. IFN- $\gamma$, TNF- $\alpha$, IL-2, IL-4, IL-5, IL-6, IL-10, IL-12p70, IL-13, IL-17A, G-CSF, and MCP-1 concentrations in the serum were determined by cytokine beads array (CBA) mouse inflammation kits (BD Biosciences) following the manufacturer's recommendation. IL-27 and IL-35 concentrations in the serum were determined by LEGEND MAX Mouse IL-27 Heterodimer ELISA Kit and LEGEND MAX Mouse IL-35 Heterodimer ELISA Kit (BioLegend), respectively. IL-22 and TGF- $\beta 1$ serum concentrations were determined by Quantikine ELISA Mouse/Rat IL-22 and Quantikine ELISA Mouse/Rat/Porcine/Canine TGF- $\beta 1$ (R\&D Systems), respectively. IFN- $\alpha$ serum concentrations were determined by the Mouse Verkine IFN- $\alpha$ ELISA Kit (PBL Assay Science). IL-35 concentrations in culture supernatants were determined by ELISA Kit for IL-35 (Cloud-Clone Corp.) following the manufacturer's recommendation.

$R T-q P C R$. Total RNA was extracted from cells using RNeasy Mini Kit or RNeasy Micro Kit (QIAGEN). Complementary DNA was synthesized by reverse transcription using the iScript cDNA Synthesis Kit (Bio-Rad). To measure the quantity, real-time PCR was performed by TaqMan Universal Master Mix with the following predesigned primers: Irf1 (Mm01288580_m1), Fas (Mm00433237_m1), Bcl2l1 (Mm00437783_m1), Il12a (Mm00434165_m1), Il12b (Mm01288992_ m1), Ebi3 (Mm00469294_m1), Il27p28 (Mm00461164_m1), Il6st (Mm00439665_m1), Il12rb1 (Mm00434189_m1), Il12rb2 (Mm00434200_m1), and Il27ra (Mm00497259_m1) using StepOne Plus systems (Applied Biosystems). The level of target gene expression was normalized against GAPDH expression in each sample.

Preparation of BM-derived PDCs. BM lymphocytes were obtained from BM cells by hemolysis and passing through a $100 \mu \mathrm{m}$ nylon mesh. BM lymphocytes $\left(1 \times 10^{6}\right.$ cells $/ \mathrm{mL}$ per 6 -well dish) were cultured in PDC conditioned medium (RPMI-1640, Sigma-Aldrich) containing 10\% FBS and 1\% penicillin/streptomycin (Nacalai Tesque), 10 mM HEPES, 1× MEM-NEAA (Gibco, Thermo Fisher Scientific), $55 \mu \mathrm{M}$ 2-mercaptoethanol (Gibco, Thermo Fisher Scientific), and 100 ng/mL mouse recombinant Flt-3L (Peprotech or Cell Guidance Systems Ltd.). After 8 days of culture, nonadherent cells were harvested and $\mathrm{B}_{2} 2 \mathrm{O}^{+}$cells were separated by magnetic beads (Miltenyi Biotec) following the manufacturer's recommendation. Over $90 \%$ purity of CD $45^{+}$CD11b-B220 ${ }^{+}$PDCA- $1^{+}$Siglec- $\mathrm{H}^{+}$cells was confirmed by flow cytometry before subsequent experiments.

In vitro Treg suppression assay. $\mathrm{CD}^{+} \mathrm{CD} 25^{-}$Teff cells and $\mathrm{CD} 4{ }^{+} \mathrm{CD} 25^{+}$Tregs were isolated from splenocytes using the $\mathrm{CD} 4{ }^{+} \mathrm{CD} 25^{+}$Regulatory $\mathrm{T}$ Cell Isolation Kit (Miltenyi Biotec) following the manufacturer's recommendation. Flt-3L-proliferated pDCs derived from WT, $I l 12 a^{-/}$, and Ebi3 ${ }^{-/}$mice were isolated as described above and further purified using cell sorting by B22O and Siglec-H staining. Over $95 \%$ purity of $\mathrm{CD}^{2} 5^{+} \mathrm{CD} 11 \mathrm{~b}-\mathrm{B} 22 \mathrm{O}^{+} \mathrm{PDCA}-1^{+}$Siglec $-\mathrm{H}^{+}$ cells was confirmed. To evaluate the effect of pDCs and/or Tregs on $\mathrm{T}$ cell proliferation, Teff cells were stained by a diluted solution of violet proliferation dye (VPD450; BD Biosciences) at $37^{\circ} \mathrm{C}$ for 15 minutes.

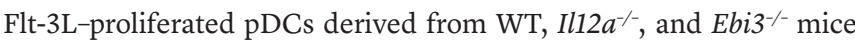
$\left(5 \times 10^{4}\right.$ cells/well $)$ were cocultured with VPD450-stained Teff cells $\left(5 \times 10^{4}\right.$ cells/well) and stimulated by $1 \mu \mathrm{L} /$ well of Dynabeads Mouse
T-Activator CD3/CD28 for T Cell Expansion and Activation (Thermo Fisher Scientific) in the presence of various numbers of Tregs (5, 2.5, $1.25 \times 10^{4}$ cells/well) at $37^{\circ} \mathrm{C}$ for 96 hours. After incubation, the cells were washed and cell proliferation was assessed by flow cytometry. $\mathrm{T}$ cell suppression was calculated according to the rate of nonproliferated Teff cells and the value of CD3/CD28; stimulated Teff cells only was considered as $0 \%$ suppression, and nonstimulated Teff cells only was considered as $100 \%$ suppression.

Statistics. Statistical analyses were performed using GraphPad Prism software version 7.0g (GraphPad). Differences between 2 groups were evaluated using 2-sided unpaired Student's $t$ tests. Comparisons of more than 2 groups were performed with 1-way ANOVA followed by Tukey-Kramer's multiple comparison test. Comparison of Kaplan-Meir curves was performed with the logrank test. For all analyses, significance was accepted at a 95\% confidence level $(P<0.05)$.

Study approval. The Animal Ethics Committee of Keio University approved all animal studies. The institutional review board of Keio University School of Medicine approved all human studies (no. 20120395, no. 20160453, and no. 20040034) according to the guidelines of the 1975 Declaration of Helsinki (2008 revision). The study subjects were prospectively recruited, and each subject provided prior written informed consent for blood sampling, study participation, and analysis of clinical data. All study subjects received standard care and treatment according to their clinical presentations. All experiments were performed according to institutional guidelines and Home Office regulations.

\section{Author contributions}

YK conceived and designed the study, performed most experiments, analyzed the data, and wrote the manuscript. NN conceived and designed the study, analyzed the data, and wrote the manuscript. PSC, AU, HT, SS, RM, and MS performed and helped with human PBMC/IHC analysis. KM and T. Sujino helped to perform experiments. YM helped to prepare the manuscript. KS and TY helped to perform experiments using genetically modified mice. YM, TT, T. Sujino, NT, AY, MS, and TY were involved in scientific and technical discussions. TK helped to conceive and supervised the study.

\section{Acknowledgments}

We thank G. Ohshima, M. Shinoda, Y. Kitagawa, and the medical staff of the surgical department of Keio University Hospital for collecting samples; H. Sato, S. Chiba, T. Amiya, R. Aoki, Y. Harada, and Y. Imura (Keio University) for technical assistance; and K. Honda and T. Tanoue (Keio University) for providing $\mathrm{IllO}^{-/-}$mice. This study was supported in part by Grants-in-Aid for Scientific Research (KAKENHI, 16K09374) from the Japan Society for the Promotion of Science and the Keio University Medical Fund. We would like to thank Editage (www.editage.jp) for English language editing.

Address correspondence to: Nobuhiro Nakamoto and Takanori Kanai, Division of Gastroenterology and Hepatology, Department of Internal Medicine, Keio University School of Medicine, Tokyo 1608582, Japan. Phone: 81.3.5363.3790; Email: nobuhiro@ z2.keio.jp; takagast@z2.keio.jp. 
1. Antoniades CG, Berry PA, Wendon JA, Vergani D. The importance of immune dysfunction in determining outcome in acute liver failure. J Hepatol. 2008;49(5):845-861.

2. Possamai LA, Thursz MR, Wendon JA, Antoniades CG. Modulation of monocyte/macrophage function: a therapeutic strategy in the treatment of acute liver failure. J Hepatol. 2014;61(2):439-445.

3. Marra F, Tacke F. Roles for chemokines in liver disease. Gastroenterology. 2014;147(3):577-594.e1.

4. Heymann F, Tacke F. Immunology in the liver-from homeostasis to disease. Nat Rev Gastroenterol Hepatol. 2016;13(2):88-110.

5. Krenkel O, Tacke F. Liver macrophages in tissue homeostasis and disease. Nat Rev Immunol. 2017;17(5):306-321.

6. Bamboat ZM, Ocuin LM, Balachandran VP, Obaid H, Plitas G, DeMatteo RP. Conventional DCs reduce liver ischemia/reperfusion injury in mice via IL-10 secretion. J Clin Invest. 2010;120(2):559-569.

7. Kawai T, Akira S. Toll-like receptors and their crosstalk with other innate receptors in infection and immunity. Immunity. 2011;34(5):637-650.

8. Gilliet M, Cao W, Liu YJ. Plasmacytoid dendritic cells: sensing nucleic acids in viral infection and autoimmune diseases. Nat Rev Immunol. 2008;8(8):594-606.

9. Swiecki M, Colonna M. The multifaceted biology of plasmacytoid dendritic cells. Nat Rev Immunol. 2015;15(8):471-485.

10. Castellaneta A, et al. Plasmacytoid dendritic cell-derived IFN- $\alpha$ promotes murine liver ischemia/ reperfusion injury by induction of hepatocyte IRF-1. Hepatology. 2014;60(1):267-277.

11. Revelo XS, et al. Nucleic acid-targeting pathways promote inflammation in obesity-related insulin resistance. Cell Rep. 2016;16(3):717-730.

12. Hannibal TD, Schmidt-Christensen A, Nilsson J, Fransén-Pettersson N, Hansen L, Holmberg D. Deficiency in plasmacytoid dendritic cells and type I interferon signalling prevents diet-induced obesity and insulin resistance in mice. Diabetologia. 2017;60(10):2033-2041.

13. Hadeiba H, Sato T, Habtezion A, Oderup C, Pan J, Butcher EC. CCR9 expression defines tolerogenic plasmacytoid dendritic cells able to suppress acute graft-versus-host disease. Nat Immunol. 2008;9(11):1253-1260.

14. Yun TJ, et al. Indoleamine 2,3-dioxygenaseexpressing aortic plasmacytoid dendritic cells protect against atherosclerosis by induction of regulatory T cells. Cell Metab. 2016;23(5):852-866.

15. Ulsenheimer A, et al. Plasmacytoid dendritic cells in acute and chronic hepatitis $\mathrm{C}$ virus infection. Hepatology. 2005;41(3):643-651.
16. Duan XZ, Wang M, Li HW, Zhuang H, Xu D, Wang FS. Decreased frequency and function of circulating plasmocytoid dendritic cells (pDC) in hepatitis B virus infected humans. J Clin Immunol. 2004;24(6):637-646.

17. Dzionek A, et al. BDCA-2, a novel plasmacytoid dendritic cell-specific type II C-type lectin, mediates antigen capture and is a potent inhibitor of interferon alpha/beta induction. J Exp Med. 2001;194(12):1823-1834.

18. Nakamoto N, et al. CCR9+ macrophages are required for acute liver inflammation in mouse models of hepatitis. Gastroenterology. 2012;142(2):366-376.

19. Zhang J, et al. Characterization of Siglec-H as a novel endocytic receptor expressed on murine plasmacytoid dendritic cell precursors. Blood. 2006;107(9):3600-3608.

20. Tokita D, et al. Poor allostimulatory function of liver plasmacytoid DC is associated with pro-apoptotic activity, dependent on regulatory T cells. J Hepatol. 2008;49(6):1008-1018.

21. Matta BM, Raimondi G, Rosborough BR, Sumpter TL, Thomson AW. IL-27 production and STAT3-dependent upregulation of B7-H1 mediate immune regulatory functions of liver plasmacytoid dendritic cells. J Immunol. 2012;188(11):5227-5237.

22. Collison LW, et al. The inhibitory cytokine IL-35 contributes to regulatory T-cell function. Nature. 2007;450(7169):566-569.

23. Collison LW, et al. IL-35-mediated induction of a potent regulatory $\mathrm{T}$ cell population. Nat Immunol. 2010;11(12):1093-1101.

24. Matta BM, Castellaneta A, Thomson AW. Tolerogenic plasmacytoid DC. Eur JImmunol. 2010;40(10):2667-2676.

25. Dasgupta S, Erturk-Hasdemir D, Ochoa-Reparaz J, Reinecker HC, Kasper DL. Plasmacytoid dendritic cells mediate anti-inflammatory responses to a gut commensal molecule via both innate and adaptive mechanisms. Cell Host Microbe. 2014;15(4):413-423.

26. Swiecki M, Wang Y, Vermi W, Gilfillan S, Schreiber RD, Colonna M. Type I interferon negatively controls plasmacytoid dendritic cell numbers in vivo. J Exp Med. 2011;208(12):2367-2374.

27. Vignali DA, Kuchroo VK. IL-12 family cytokines: immunological playmakers. Nat Immunol. 2012;13(8):722-728.

28. Shen $\mathrm{P}$, et al. IL-35-producing B cells are critical regulators of immunity during autoimmune and infectious diseases. Nature. 2014;507(7492):366-370.

29. Shamji MH, et al. Role of IL-35 in sublingual allergen immunotherapy. JAllergy Clin Immunol. 2019;143(3):1131-1142.e4

30. Wang RX, et al. Interleukin-35 induces regulatory $\mathrm{B}$ cells that suppress autoimmune disease. Nat Med. 2014;20(6):633-641.

31. Dambuza IM, et al. IL-12p35 induces expansion of IL-10 and IL-35-expressing regulatory B cells and ameliorates autoimmune disease. Nat Commun. 2017;8(1):719.

32. Dixon KO, van der Kooij SW, Vignali DA, van Kooten C. Human tolerogenic dendritic cells produce IL-35 in the absence of other IL-12 family members. Eur J Immunol. 2015;45(6):1736-1747.

33. Lee CC, et al. Macrophage-secreted interleukin-35 regulates cancer cell plasticity to facilitate metastatic colonization. Nat Commun. 2018;9(1):3763.

34. Ryo K, et al. Significance of Fas antigen-mediated apoptosis in human fulminant hepatic failure. $\mathrm{Am}$ J Gastroenterol. 2000;95(8):2047-2055.

35. Luedde T, Kaplowitz N, Schwabe RF. Cell death and cell death responses in liver disease: mechanisms and clinical relevance. Gastroenterology. 2014;147(4):765-783.e4.

36. Crispe IN. The liver as a lymphoid organ. Annu Rev Immunol. 2009;27:147-163.

37. Dong Z, Wei H, Sun R, Tian Z. The roles of innate immune cells in liver injury and regeneration. Cell Mol Immunol. 2007;4(4):241-252.

38. Wei HX, et al. CD4+ CD25+ Foxp3+ regulatory $\mathrm{T}$ cells protect against $\mathrm{T}$ cell-mediated fulminant hepatitis in a TGF-beta-dependent manner in mice. J Immunol. 2008;181(10):7221-7229.

39. Manns MP, Lohse AW, Vergani D. Autoimmune hepatitis--update 2015. J Hepatol. 2015;62(1 Suppl):S100-S111.

40. Iwai M, Jo M, Ishii M, Mori T, Harada Y. Comparison of clinical features and liver histology in acute and chronic autoimmune hepatitis. Hepatol Res. 2008;38(8):784-789.

41. Weiler-Normann C, Lohse AW. Acute autoimmune hepatitis: many open questions. J Hepatol. 2014;61(4):727-729.

42. Takagi H, et al. Plasmacytoid dendritic cells are crucial for the initiation of inflammation and $\mathrm{T}$ cell immunity in vivo. Immunity. 2011;35(6):958-971.

43. Kawai T, Adachi O, Ogawa T, Takeda K, Akira S. Unresponsiveness of MyD88-deficient mice to endotoxin. Immunity. 1999;11(1):115-122.

44. Nakamoto N, et al. Commensal lactobacillus controls immune tolerance during acute liver injury in mice. Cell Rep. 2017;21(5):1215-1226.

45. Taniki N, et al. Intestinal barrier regulates immune responses in the liver via IL-10-producing macrophages. JCI Insight. 2018;3(12):91980. 